\title{
Gas-Phase Hydration of Perillaldehyde Investigated by Microwave Spectroscopy
} Assisted by Computational Chemistry Mhamad Chrayteh, Thérèse R. Huet and Pascal Dréan University of Lille, CNRS, UMR 8523 - PhLAM - Physique des Lasers, Atomes et Molécules, F-59000 Lille, France 


\section{Contents}

\section{List of Figures}

S1 The structures of the monohydrates of perillaldehyde $1 \mathrm{w}(\mathrm{I})$ and $1 \mathrm{w}(\mathrm{II})$. The values in blue are relative to the hydrogen bond. The shortest distances between oxygen atoms of water and hydrogen atoms of perillaldehyde are indicated in purple, and the angles are shown in red. Lengths are expressed in $\AA$ and angles in ${ }^{\circ}$. . . . . . . . . . . . . . . . . S6

S2 NCI plots showing the interactions in the monohydrates of perillaldehyde. . . . . . . . S7

S3 The structures of the dihydrates of perillaldehyde $2 \mathrm{w}(\mathrm{I})$ and $2 \mathrm{w}(\mathrm{II})$. The values in blue are relative to the hydrogen bond. The shortest distances between oxygen atoms of water and hydrogen atoms of perillaldehyde are indicated in purple, and the angles are shown in red. Lengths are expressed in $\AA$ and angles in ${ }^{\circ}$. . . . . . . . . . . . . . . . . . . S11

S4 NCI plots showing the interactions in the dihydrates of perillaldehyde. The parameters used are $(s=0.5)$ and $\operatorname{sign}\left(\lambda_{2}\right) \rho$ between $-0.05 \mathrm{a} u$ and $+0.05 \mathrm{a}$. . . . . . . . . . . S12

S5 The structures of the trihydrates $3 \mathrm{w}(\mathrm{I})$ with the two parent molecules of perillaldehyde. The values in blue are relative to the hydrogen bond. The shortest distances between oxygen atoms of water and hydrogen atoms of perillaldehyde are indicated in purple, and the angles are shown in red. Lengths are expressed in $\AA$ and angles in ${ }^{\circ}$. . . . . . . . S17

S6 NCI plots showing the interactions in the trihydrates of perillaldehyde. The parameters used are $(s=0.5)$ and $\operatorname{sign}\left(\lambda_{2}\right) \rho$ between $-0.05 \mathrm{a} u$ and $+0.05 \mathrm{a} u \ldots \ldots$. . . . . . S19

S7 An example of a structured line of a monohydrate of perillaldehyde. The splitting of $3.7 \mathrm{kHz}$ in this case can be clearly seen. . . . . . . . . . . . . . . . . S35

S8 Potential energy curves of the methyl group rotation around the $\mathrm{C}_{8}-\mathrm{C}_{9}$ bond calculated at the B3LYP-D3BJ/6-311++G(d,p) level using Gaussian 16 in perillaldehyde and in its hydrates. All structural parameters were allowed to be optimized, except the $\tau\left(\mathrm{H}_{10} \mathrm{C}_{9} \mathrm{C}_{8} \mathrm{C}_{7}\right)$ dihedral angle which was incremented with a step of $5^{\circ} . \ldots$. . . . S36

\section{List of Tables}

S1 Calculated rotational constants, dipole moments and relative energies (including ZPE corrections) of the 4 monohydrates of perillaldehyde. 
S2 Experimental rotational and quartic centrifugal distortion constants of the normal and of the $\mathrm{H}_{2}^{18} \mathrm{O}$ substituted species of the Eq1-1w(I) hydrate of perillaldehyde. . . . . . . . . .

S3 Experimental rotational and quartic centrifugal distortion constants of the normal and of the $\mathrm{H}_{2}^{18} \mathrm{O}$ substituted species of the Eq1-1w(II) hydrate of perillaldehyde.

S4 Experimental rotational and quartic centrifugal distortion constants of the normal and of the $\mathrm{H}_{2}^{18} \mathrm{O}$ substituted species of the EQ2-1w(I) hydrate of perillaldehyde.

S5 Experimental rotational and quartic centrifugal distortion constants of the normal and of the $\mathrm{H}_{2}^{18} \mathrm{O}$ substituted species of the EQ2-1w(II) hydrate of perillaldehyde. . . . . . . . . S10

S6 Calculated rotational constants, dipole moments and relative energies (including ZPE corrections) of the four dihydrates of perillaldehyde. .

S7 Experimental rotational and quartic centrifugal distortion constants of the normal and of the $\mathrm{H}_{2}^{18} \mathrm{O}$ substituted species of the EQ1-2w(I) hydrate of perillaldehyde. . . . . . . . . S14

S8 Experimental rotational and quartic centrifugal distortion constants of the normal and of the $\mathrm{H}_{2}^{18} \mathrm{O}$ substituted species of the EQ2-2w(I) hydrate of perillaldehyde. . . . . . . . . S15

S9 Experimental second planar moments (in $\mathrm{u} \AA^{2}$ ) of the hydrates of perillaldehyde and of their isotopic species. . . . . . . . . . . . . . . . . . . . S16

S10 Measured frequencies $\left(v_{\text {obs. }}\right)$ and residuals $\left(v_{\text {obs. }}-v_{\text {calc. }}\right)$ in $\mathrm{MHz}$ of the monohydrate of perillaldehyde EQ1-1w(I) . . . . . . . . . . . . . . . . . . . . S18

S11 Measured frequencies $\left(v_{\text {obs. }}\right)$ and residuals $\left(v_{\text {obs. }}-v_{\text {calc. }}\right)$ in $\mathrm{MHz}$ of the monohydrate of perillaldehyde EQ1-1w(I) labeled with ${ }^{18} \mathrm{O} \ldots \ldots \ldots$. . . . . . . . . . . . . . S20

S12 Measured frequencies $\left(v_{\text {obs. }}\right)$ and residuals $\left(v_{\text {obs. }}-v_{\text {calc. }}\right)$ in $\mathrm{MHz}$ of the monohydrate of perillaldehyde EQ1-1w(II) . . . . . . . . . . . . . . . . . . . . . . S21

S13 Measured frequencies $\left(v_{\text {obs. }}\right)$ and residuals $\left(v_{\text {obs. }}-v_{\text {calc. }}\right)$ in $\mathrm{MHz}$ of the monohydrate of perillaldehyde EQ1-1w(II) labeled with ${ }^{18} \mathrm{O} \ldots \ldots \ldots \ldots$. . . . . . . . . . . S22

S14 Measured frequencies ( $\left.v_{\text {obs. }}\right)$ and residuals $\left(v_{\text {obs. }}-v_{\text {calc. }}\right)$ in $\mathrm{MHz}$ of the monohydrate of perillaldehyde EQ2-1w(I).

S15 Measured frequencies $\left(v_{\text {obs. }}\right)$ and residuals $\left(v_{\text {obs. }}-v_{\text {calc. }}\right)$ in $\mathrm{MHz}$ of the monohydrate of perillaldehyde EQ2-1w(I) labeled with ${ }^{18} \mathrm{O} \ldots \ldots \ldots$. . . . . . . . . . . . S25

S16 Measured frequencies $\left(v_{\text {obs. }}\right)$ and residuals $\left(v_{\text {obs. }}-v_{\text {calc. }}\right)$ in $\mathrm{MHz}$ of the monohydrate of perillaldehyde EQ2-1w(II) . . . . . . . . . . . . . . . . . . . . . S26 
S17 Measured frequencies $\left(v_{\text {obs. }}\right)$ and residuals $\left(v_{\text {obs. }}-v_{\text {calc. }}\right)$ in $\mathrm{MHz}$ of the monohydrate of perillaldehyde EQ2-1w(II) labeled with ${ }^{18} \mathrm{O} \ldots \ldots \ldots$. . . . . . . . . . . . . . . S27

S18 Measured frequencies $\left(v_{\text {obs. }}\right)$ and residuals $\left(v_{\text {obs. }}-v_{\text {calc. }}\right)$ in $\mathrm{MHz}$ of the dihydrate of perillaldehyde EQ1-2w(I) . . . . . . . . . . . . . . . . . . . . . . S28

S19 Measured frequencies $\left(v_{\text {obs. }}\right)$ and residuals $\left(v_{\text {obs. }}-v_{\text {calc. }}\right)$ in $\mathrm{MHz}$ of the dihydrate of perillaldehyde EQ1-2w(I) with the ${ }^{16} \mathrm{O}_{\mathrm{w} 1}$ atom substituted by a ${ }^{18} \mathrm{O}$ atom. . . . . . . . . . . . S30

S20 Measured frequencies $\left(v_{\text {obs. }}\right)$ and residuals $\left(v_{\text {obs. }}-v_{\text {calc. }}\right)$ in $\mathrm{MHz}$ of the dihydrate of perillaldehyde EQ1-2w(I) with the ${ }^{16} \mathrm{O}_{\mathrm{w} 2}$ atom substituted by a ${ }^{18} \mathrm{O}$ atom. . . . . . . . . . . . S30

S21 Measured frequencies $\left(v_{\text {obs. }}\right)$ and residuals $\left(v_{\text {obs. }}-v_{\text {calc. }}\right)$ in $\mathrm{MHz}$ of the dihydrate of perillaldehyde EQ1-2w(I) with the ${ }^{16} \mathrm{O}_{\mathrm{w} 1}$ and ${ }^{16} \mathrm{O}_{\mathrm{w} 2}$ atoms substituted by ${ }^{18} \mathrm{O}$ atoms. . . . . . S31

S22 Measured frequencies $\left(v_{\text {obs. }}\right)$ and residuals $\left(v_{\text {obs. }}-v_{\text {calc. }}\right)$ in $\mathrm{MHz}$ of the dihydrate of perillaldehyde EQ2-2w(I) . . . . . . . . . . . . . . . . . . . . . . . . S32

S23 Measured frequencies $\left(v_{\text {obs. }}\right)$ and residuals $\left(v_{\text {obs. }}-v_{\text {calc. }}\right)$ in $\mathrm{MHz}$ of the dihydrate of perillaldehyde EQ2-2w(I) with the ${ }^{16} \mathrm{O}_{\mathrm{w} 1}$ atom substituted by a ${ }^{18} \mathrm{O}$ atom. . . . . . . . . . . . S33

S24 Measured frequencies $\left(v_{\text {obs. }}\right)$ and residuals $\left(v_{\text {obs. }}-v_{\text {calc. }}\right)$ in $\mathrm{MHz}$ of the dihydrate of perillaldehyde EQ2-2w(I) with the ${ }^{16} \mathrm{O}_{\mathrm{w} 2}$ atom substituted by a ${ }^{18} \mathrm{O}$ atom. . . . . . . . . . . . S34

S25 Measured frequencies $\left(v_{\text {obs. }}\right)$ and residuals $\left(v_{\text {obs. }}-v_{\text {calc. }}\right)$ in $\mathrm{MHz}$ of the dihydrate of perillaldehyde EQ2-2w(I) with the ${ }^{16} \mathrm{O}_{\mathrm{w} 1}$ and ${ }^{16} \mathrm{O}_{\mathrm{w} 2}$ atoms substituted by ${ }^{18} \mathrm{O}$ atoms. . . . . . S34

S26 The computed rotational constants and dipole moment components of the two trihydrates of perillaldehyde . . . . . . . . . . . . . . . . . . S35 


\section{Complete references}

(3) Mentel, T. F.; Wildt, J.; Kiendler-Scharr, A.; Kleist, E.; Tillmann, R.; Dal Maso, M.; Fisseha, R.; Hohaus, T.; Spahn, H.; Uerlings, R.; Wegener, R.; Griffiths, P. T.; Dinar, E.; Rudich, Y.; Wahner, A. Photochemical Production of Aerosols From Real Plant Emissions J. Atmos. Chem. Phys. 2009, 9, 4387-4406.

(5) Baltensperger, U.; Kalberer, M.; Dommen, J.; Paulsen, D.; Alfarra, M. R.; Coe, H.; Fisseha, R.; Gascho, A.; Gysel, M.; Nyeki, S.; Sax, M.; Steinbacher, M.; Prevot, A.S.H.; Sjögren, S.; Weingartner, E.; Zenobi, R. Secondary Organic Aerosols from Anthropogenic and Biogenic Precursors Faraday Discuss. 2005, 130, 265-278.

(9) Stirnweis, L.; Marcolli, C.; Dommen, J.; Barmet, P.; Frege, C.; Platt, S.; Bruns, E.; Krapf, M.; Slowik, J.; Wolf, R.; Prévôt, A.; Baltensperger, U.; El-Haddad, I. Assessing the Influence of NOx Concentrations and Relative Humidity on Secondary Organic Aerosol Yields from $\alpha$-Pinene Photo-Oxidation Through Smog Chamber Experiments and Modelling Calculations Atmos. Chem. Phys. 2017,17, 50355061.

(22) Masumoto, N.; Nishizaki, Y.; Maruyama, T.; Igarashi, Y.; Nakajima, K.; Yamazaki, T.; Kuroe, M.; Numata, M.; Ihara, T.; Sugimoto, N.; Sato, K. Determination of Perillaldehyde in Perilla Herbs Using Relative Molar Sensitivity to Single-Reference Diphenyl Sulfone J. Nat. Med. 2019, 73, 566-576.

(31) Frisch, M. J.; Trucks, G. W.; Schlegel, H. B.; Scuseria, G. E.; Robb, M. A.; Cheeseman, J. R.; Scalmani, G.; Barone, V.; Petersson, G. A.; Nakatsuji, H.; Li, X.; Caricato, M.; Marenich, A. V.; Bloino, J.; Janesko, B. G.; Gomperts, R.; Mennucci, B.; Hratchian, H. P.; Ortiz, J. V.; Izmaylov, A. F.; Sonnenberg, J. L.; Williams-Young, D.; Ding, F.; Lipparini, F.; Egidi, F.; Goings, J.; Peng, B.; Petrone, A.; Henderson, T.; Ranasinghe, D.; Zakrzewski, V. G.; Gao, J.; Rega, N.; Zheng, G.; Liang, W.; Hada, M.; Ehara, M.; Toyota, K.; Fukuda, R.; Hasegawa, J.; Ishida, M.; Nakajima, T.; Honda, Y.; Kitao, O.; Nakai, H.; Vreven, T.; Throssell, K.; Montgomery Jr., J. A.; Peralta, J. E.; Ogliaro, F.; Bearpark, M. J.; Heyd, J. J.; Brothers, E. N.; Kudin, K. N.; Staroverov, V. N.; Keith, T. A.; Kobayashi, R.; Normand, J.; Raghavachari, K.; Rendell, A. P.; Burant, J. C.; Iyengar, S. S.; Tomasi, J.; Cossi, M.; Millam, J. M.; Klene, M.; Adamo, C.; Cammi, R.; Ochterski, J. W.; Martin, R. L.; Morokuma, K.; Farkas, O.; Foresman, J. B.; Fox, D. J. Gaussian 16 Revision B.01, Gaussian Inc. Wallingford CT, 2016.

(45) Parrish, R.; Burns, L.; Smith, D.; Simmonett, A.; DePrince, A.; Hohenstein, E.; Bozkaya, U.; Sokolov, A.; Di Remigio, R.; Richard, R.; Gonthier, J.; James, A.; McAlexander, H.; Kumar, A.; Saitow, M.; Wang, X.; Pritchard, B.; Verma, P.; Schaefer, H.; Patkowski, K.; King, R.; Valeev, E.; Evangelista, F.; Turney, J.; Crawford, T.; Sherrill, C. Psi4 1.1: An Open-Source Electronic Structure Program Emphasizing Automation, Advanced Libraries, and Interoperability J. Chem. Theory Comput. 2017, 13, 3185-3197. 


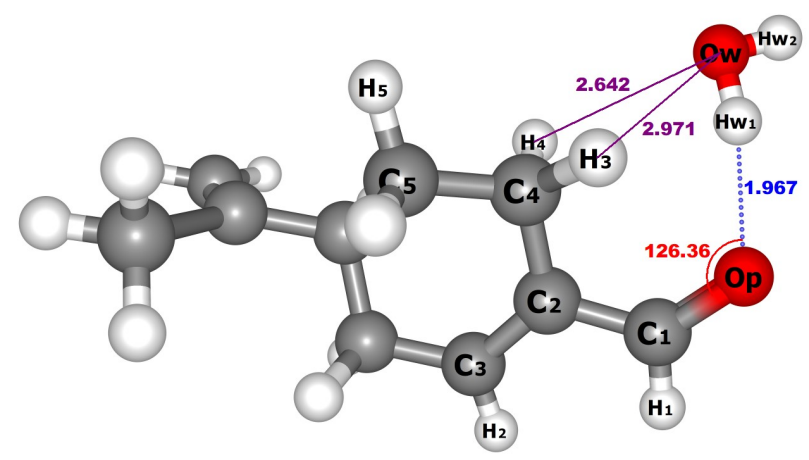

(a) EQ1-1w(I)

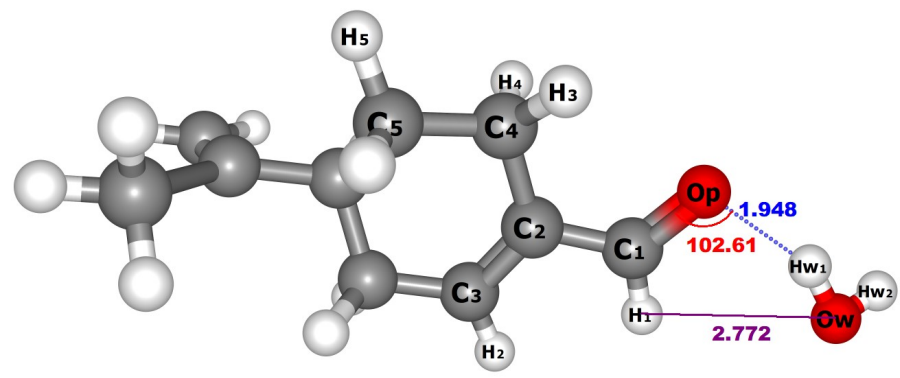

(c) EQ1-1w(II) $\left(2.02 \mathrm{~kJ} \mathrm{~mol}^{-1}\right)$

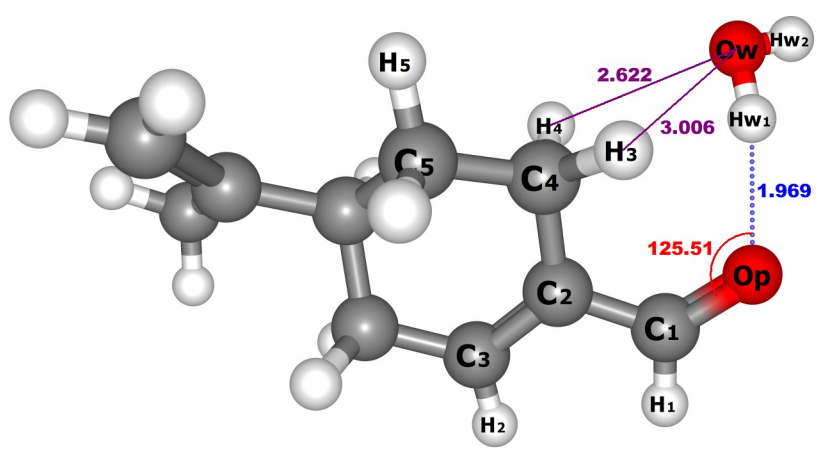

(b) EQ2-1w(I) $\left(2.23 \mathrm{~kJ} \mathrm{~mol}^{-1}\right)$

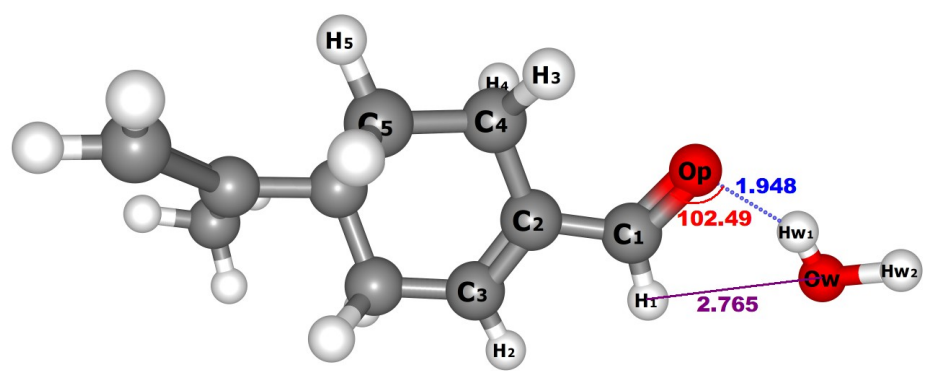

(d) EQ2-1w(II) $\left(4.26 \mathrm{~kJ} \mathrm{~mol}^{-1}\right)$

Figure S1: The structures of the monohydrates of perillaldehyde $1 \mathrm{w}(\mathrm{I})$ and $1 \mathrm{w}(\mathrm{II})$. The values in blue are relative to the hydrogen bond. The shortest distances between oxygen atoms of water and hydrogen atoms of perillaldehyde are indicated in purple, and the angles are shown in red. Lengths are expressed in $\AA$ and angles in . 


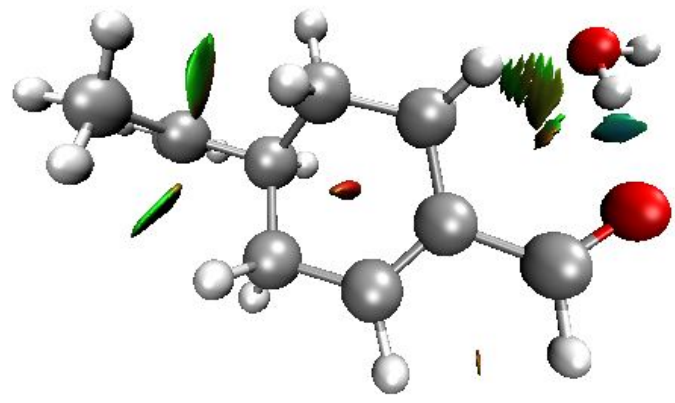

(a) EQ1-1w(I)

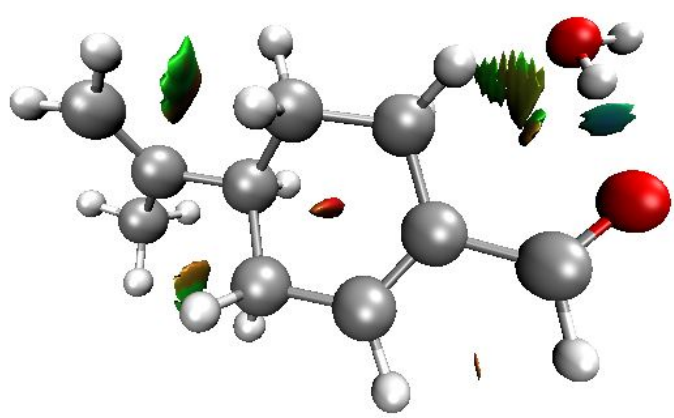

(c) EQ2-1w(I)

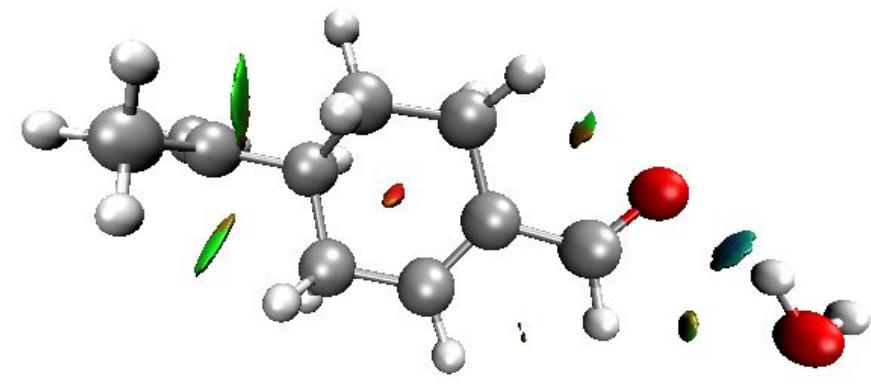

(b) EQ1-1w(II)

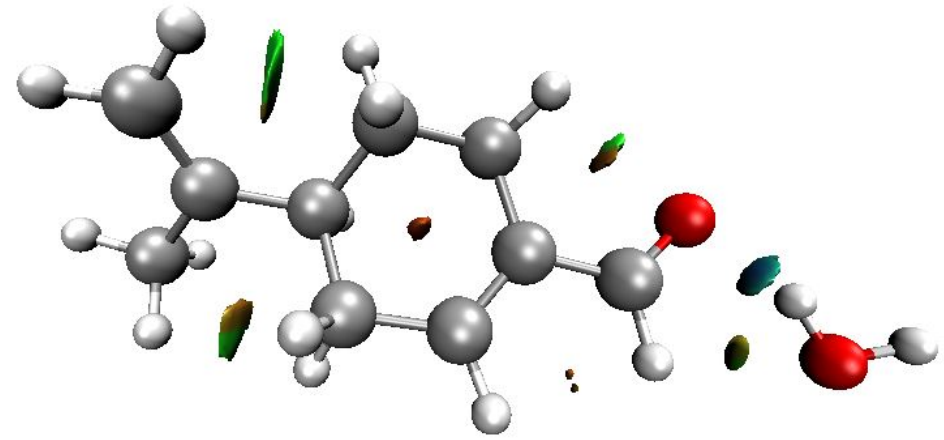

(d) EQ2-1w(II)
Strong attractive interaction (hydrogen bond)
Weak interactions (dispersion, Van der Waals)
Strong repulsive interactions (steric)

Figure S2: NCI plots showing the interactions in the monohydrates of perillaldehyde. 
Table S1: Calculated rotational constants, dipole moments and relative energies (including ZPE corrections) of the 4 monohydrates of perillaldehyde.

\begin{tabular}{|c|c|c|c|c|}
\hline Parameter & $\mathrm{DFT}^{a}$ & $\mathrm{MP}^{b}$ & $\mathrm{DFT}^{a}$ & $\mathrm{MP} 2^{b}$ \\
\hline & \multicolumn{2}{|c|}{ EQ1-1w(I) } & \multicolumn{2}{|c|}{ EQ1-1w(II) } \\
\hline$A / \mathrm{MHz}$ & 1646.92 & 1607.75 & 2810.90 & 2763.57 \\
\hline$B / \mathrm{MHz}$ & 423.64 & 427.21 & 317.30 & 317.98 \\
\hline $\mathrm{C} / \mathrm{MHz}$ & 368.71 & 373.92 & 306.35 & 306.74 \\
\hline$\left|\mu_{a}\right| / \mathrm{D}$ & 2.50 & 2.04 & 4.18 & 3.37 \\
\hline$\left|\mu_{b}\right| / \mathrm{D}$ & 2.64 & 2.52 & 0.50 & 0.12 \\
\hline$\left|\mu_{c}\right| / \mathrm{D}$ & 0.30 & 0.14 & 0.69 & 0.44 \\
\hline \multirow[t]{2}{*}{$\Delta E^{c} / \mathrm{kJ} \mathrm{mol}^{-1}$} & 0.0 & 0.0 & 1.73 & 2.02 \\
\hline & \multicolumn{2}{|c|}{ EQ2-1w(I) } & \multicolumn{2}{|c|}{ EQ2-1w(II) } \\
\hline$A / \mathrm{MHz}$ & 1675.21 & 1630.14 & 2784.82 & 2721.22 \\
\hline$B / \mathrm{MHz}$ & 422.85 & 423.89 & 321.35 & 322.70 \\
\hline $\mathrm{C} / \mathrm{MHz}$ & 362.86 & 370.55 & 299.37 & 299.94 \\
\hline$\left|\mu_{a}\right| / \mathrm{D}$ & 2.62 & 2.12 & 4.17 & 3.31 \\
\hline$\left|\mu_{b}\right| / \mathrm{D}$ & 2.76 & 2.62 & 0.31 & 0.03 \\
\hline$\left|\mu_{c}\right| / \mathrm{D}$ & 0.06 & 0.35 & 1.04 & 0.71 \\
\hline$\Delta E^{c} / \mathrm{kJ} \mathrm{mol}^{-1}$ & 1.44 & 2.23 & 3.11 & 4.26 \\
\hline
\end{tabular}

${ }^{a}$ B3LYP-D3BJ/def2-TZVP $;{ }^{b} \mathrm{MP} 2$ / 6-311++G(d,p) ; ${ }^{c}$ Energy gap relative to conformer EQ1-1w(I) (including ZPE correction). 
Table S2: Experimental rotational and quartic centrifugal distortion constants of the normal and of the $\mathrm{H}_{2}^{18} \mathrm{O}$ substituted species of the Eq1-1w(I) hydrate of perillaldehyde.

\begin{tabular}{lcccc}
\hline & Normal & $\mathrm{MP2}^{a}$ & $\mathrm{DFT}^{b}$ & ${ }^{18} \mathrm{O}_{1}$ \\
\hline $\mathrm{A} / \mathrm{MHz}$ & $1614.3942(12)$ & 1607.75 & 1646.92 & $1565.811(11)$ \\
$B / \mathrm{MHz}$ & $418.38003(13)$ & 427.21 & 423.64 & $408.54132(14)$ \\
$\mathrm{C} / \mathrm{MHz}$ & $363.12792(11)$ & 373.92 & 368.71 & $353.28895(11)$ \\
$\Delta_{J} / \mathrm{kHz}$ & $0.03849(41)$ & 0.030 & 0.040 & $0.04068(56)$ \\
$\Delta_{J K} / \mathrm{kHz}$ & $0.1653(34)$ & 0.201 & 0.344 & $0.155(17)$ \\
$\Delta_{K} / \mathrm{kHz}$ & - & 0.514 & 0.271 & - \\
$\delta_{J} / \mathrm{kHz}$ & $0.00394(31)$ & 0.001 & -0.001 & $0.00500(50)$ \\
$\delta_{K} / \mathrm{kHz}$ & $-0.345(27)$ & -0.103 & -0.7258 & $c$ \\
$\sigma / \mathrm{kHz}$ & 1.11 & - & - & 1.04 \\
$N_{\text {lines }}$ & 45 & - & - & 23 \\
$\kappa$ & -0.912 & -0.914 & -0.914 & -0.909 \\
\hline
\end{tabular}

${ }^{a} \mathrm{MP} 2 / 6-311++\mathrm{G}(\mathrm{d}, \mathrm{p}){ }^{b}{ }^{b}$ B 3LYP-D3BJ /def2-TZVP; ${ }^{c}$ Fixed at the value of the parent species.

Table S3: Experimental rotational and quartic centrifugal distortion constants of the normal and of the $\mathrm{H}_{2}^{18} \mathrm{O}$ substituted species of the Eq1-1w(II) hydrate of perillaldehyde.

\begin{tabular}{lcccc}
\hline & Normal & $\mathrm{MP2}^{a}$ & $\mathrm{DFT}^{b}$ & $1^{2} \mathrm{O}_{1}$ \\
\hline$A / \mathrm{MHz}$ & $2772.57(47)$ & 2763.57 & 2784.82 & $2765.52(59)$ \\
$B / \mathrm{MHz}$ & $316.691323(72)$ & 317.98 & 321.35 & $304.451234(60)$ \\
$C / \mathrm{MHz}$ & $305.593637(66)$ & 306.74 & 299.36 & $294.076752(58)$ \\
$\Delta_{J} / \mathrm{kHz}$ & $0.01571(32)$ & 0.015 & 0.014 & $0.01741(32)$ \\
$\Delta_{J K} / \mathrm{kHz}$ & $-0.174(18)$ & -0.174 & -0.240 & $-0.283(23)$ \\
$\Delta_{K} / \mathrm{kHz}$ & - & 2.440 & 3.338 & $c$ \\
$\delta_{J} / \mathrm{kHz}$ & - & 0.001 & 0.002 & $c$ \\
$\delta_{K} / \mathrm{kHz}$ & - & -0.290 & 0.16 & $c$ \\
$\sigma / \mathrm{kHz}$ & 1.57 & - & - & 0.93 \\
$N_{\text {lines }}$ & 30 & - & - & 18 \\
$\kappa$ & -0.991 & -0.991 & -0.982 & -0.992 \\
\hline
\end{tabular}

${ }^{a} \mathrm{MP} 2 / 6-311++\mathrm{G}(\mathrm{d}, \mathrm{p}){ }^{b}{ }^{b} \mathrm{~B} 3 \mathrm{LYP}-\mathrm{D} 3 \mathrm{BJ} / \mathrm{def} 2-\mathrm{TZVP} ;{ }^{c}$ Fixed at the value of the parent species. 
Table S4: Experimental rotational and quartic centrifugal distortion constants of the normal and of the $\mathrm{H}_{2}^{18} \mathrm{O}$ substituted species of the EQ2-1w(I) hydrate of perillaldehyde.

\begin{tabular}{lcccc}
\hline & Normal & $\mathrm{MP2}^{a}$ & $\mathrm{DFT}^{b}$ & ${ }^{18} \mathrm{O}_{1}$ \\
\hline$A / \mathrm{MHz}$ & $1646.3269(15)$ & 1630.14 & 1675.21 & $1597.7392(54)$ \\
$B / \mathrm{MHz}$ & $417.88068(13)$ & 423.89 & 422.85 & $407.740142(53)$ \\
$C / \mathrm{MHz}$ & $356.34154(12)$ & 370.55 & 362.86 & $346.912218(73)$ \\
$\Delta_{J} / \mathrm{kHz}$ & $0.04454(39)$ & 0.035 & 0.043 & $0.04704(31)$ \\
$\Delta_{J K} / \mathrm{kHz}$ & $0.1428(56)$ & 0.185 & 0.263 & $0.143(12)$ \\
$\Delta_{K} / \mathrm{kHz}$ & - & 0.654 & 0.568 & - \\
$\delta_{J} / \mathrm{kHz}$ & $-0.00204(29)$ & -0.003 & -0.007 & $c$ \\
$\delta_{K} / \mathrm{kHz}$ & $0.321(35)$ & 0.390 & 0.536 & $c$ \\
$\sigma / \mathrm{kHz}$ & 1.29 & - & - & 0.94 \\
$N_{\text {lines }}$ & 42 & - & - & 23 \\
$\kappa$ & -0.904 & -0.915 & -0.909 & -0.903 \\
\hline
\end{tabular}

${ }^{a} \mathrm{MP} 2 / 6-311++\mathrm{G}(\mathrm{d}, \mathrm{p}) ;{ }^{b}$ B3LYP-D3BJ/def2-TZVP; ${ }^{c}$ Fixed at the value of the parent species.

Table S5: Experimental rotational and quartic centrifugal distortion constants of the normal and of the $\mathrm{H}_{2}^{18} \mathrm{O}$ substituted species of the EQ2-1w(II) hydrate of perillaldehyde.

\begin{tabular}{lcccc}
\hline & Normal & $\mathrm{MP2}^{a}$ & $\mathrm{DFT}^{b}$ & $1^{18} \mathrm{O}_{1}$ \\
\hline$A / \mathrm{MHz}$ & $2749.841(39)$ & 2721.22 & 2784.82 & $2738.32(15)$ \\
$B / \mathrm{MHz}$ & $320.67503(10)$ & 322.70 & 321.34 & $308.143249(67)$ \\
$C / \mathrm{MHz}$ & $298.686140(72)$ & 299.94 & 299.36 & $287.663130(60)$ \\
$\Delta_{J} / \mathrm{kHz}$ & $0.01676(13)$ & 0.016 & 0.014 & $0.01671(20)$ \\
$\Delta_{J K} / \mathrm{kHz}$ & $-0.3346(80)$ & -0.323 & -0.240 & $-0.297(45)$ \\
$\Delta_{K} / \mathrm{kHz}$ & - & 4.287 & 3.338 & - \\
$\delta_{J} / \mathrm{kHz}$ & $0.00215(17)$ & 0.0021 & 0.0018 & $0.00221(14)$ \\
$\delta_{K} / \mathrm{kHz}$ & - & 0.1562 & 0.1602 & - \\
$\sigma / \mathrm{kHz}$ & 0.81 & - & - & 0.39 \\
$N_{\text {lines }}$ & 29 & - & - & 17 \\
$\kappa$ & -0.982 & -0.981 & -0.982 & -0.983 \\
\hline
\end{tabular}

${ }^{a} \mathrm{MP2} /$ 6-311++G(d,p); ${ }^{b}$ B3LYP-D3BJ/def2-TZVP. 


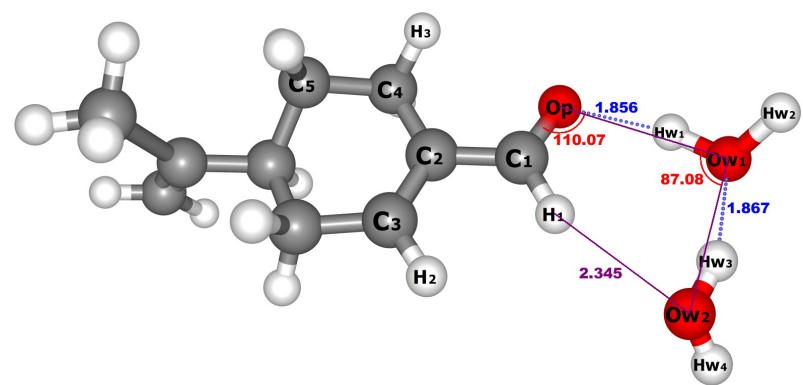

(a) EQ1-2w(I)

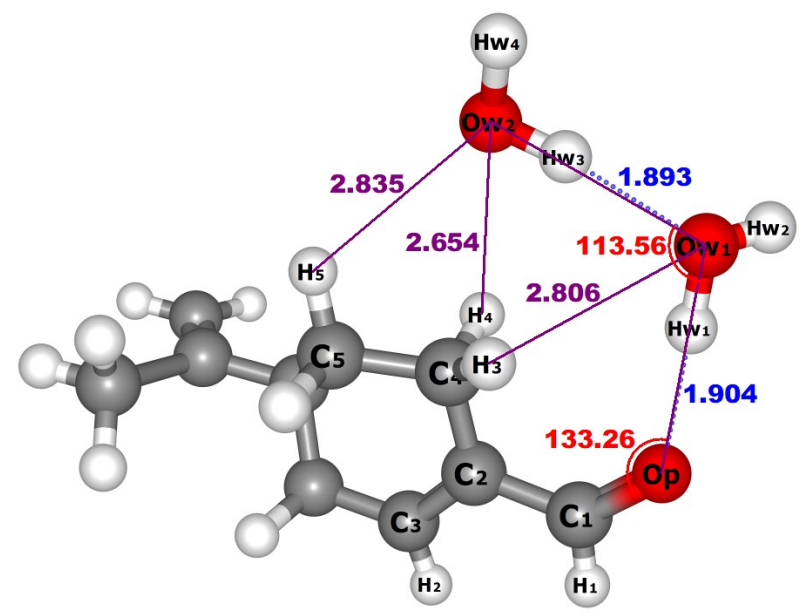

(c) EQ1-2w(II) $\left(0.98 \mathrm{~kJ} \mathrm{~mol}^{-1}\right)$

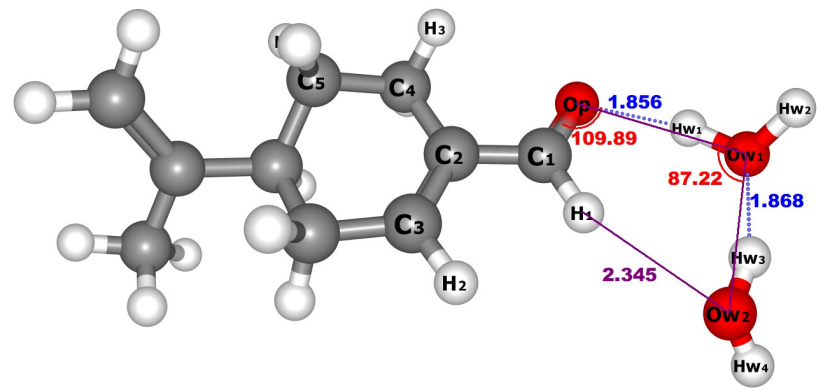

(b) EQ2-2w(I) $\left(1.35 \mathrm{~kJ} \mathrm{~mol}^{-1}\right)$

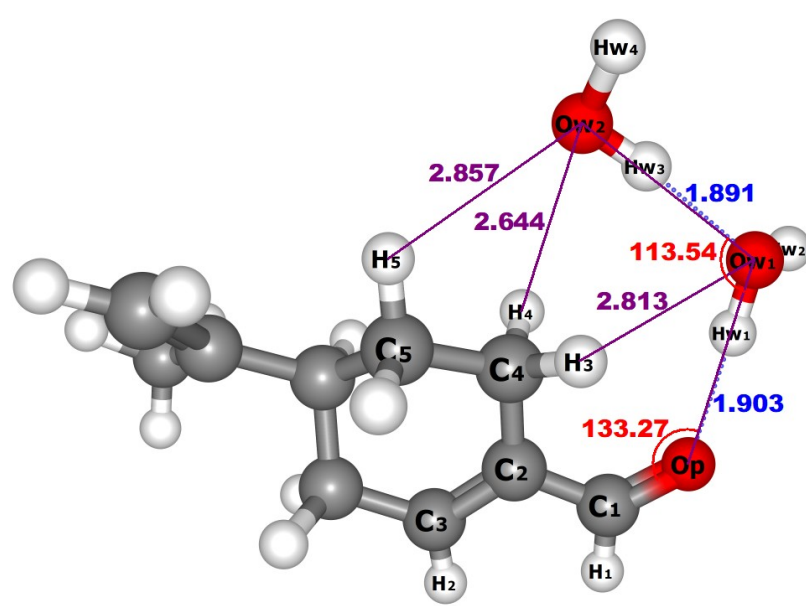

(d) EQ2-1w(II) $\left(1.99 \mathrm{~kJ} \mathrm{~mol}^{-1}\right)$

Figure S3: The structures of the dihydrates of perillaldehyde $2 \mathrm{w}(\mathrm{I})$ and $2 \mathrm{w}(\mathrm{II})$. The values in blue are relative to the hydrogen bond. The shortest distances between oxygen atoms of water and hydrogen atoms of perillaldehyde are indicated in purple, and the angles are shown in red. Lengths are expressed in $\AA$ and angles in ${ }^{\circ}$. 


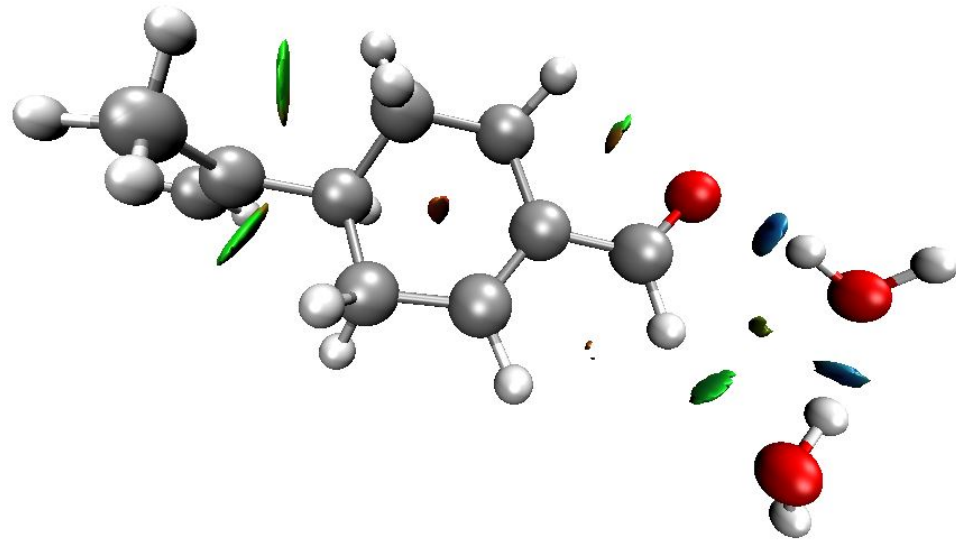

(a) EQ1-2w(I)

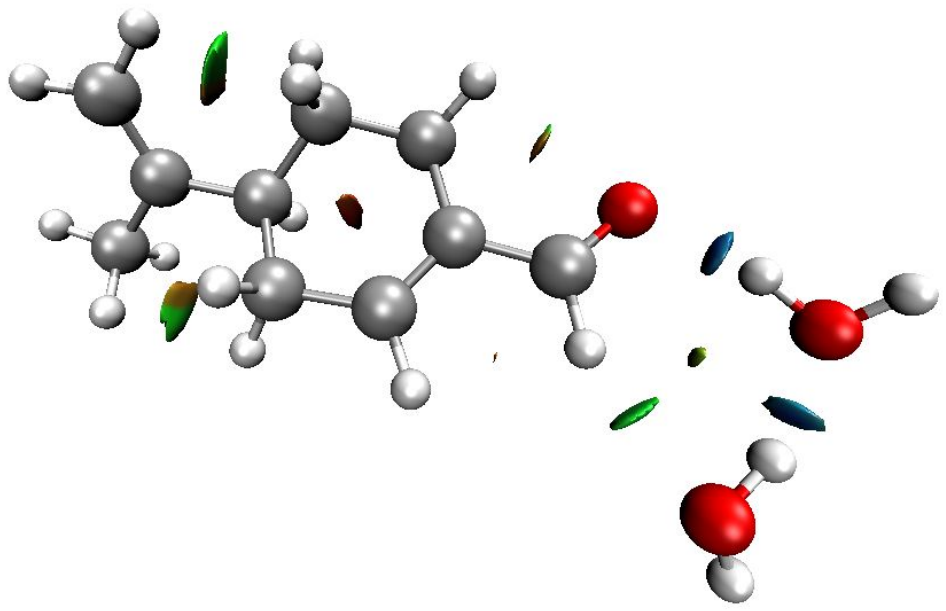

(c) EQ2-2w(I)

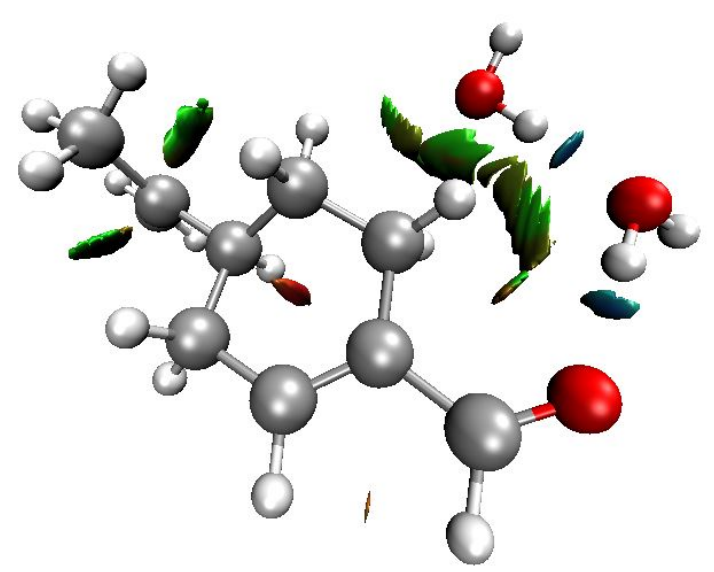

(b) EQ1-2w(II)

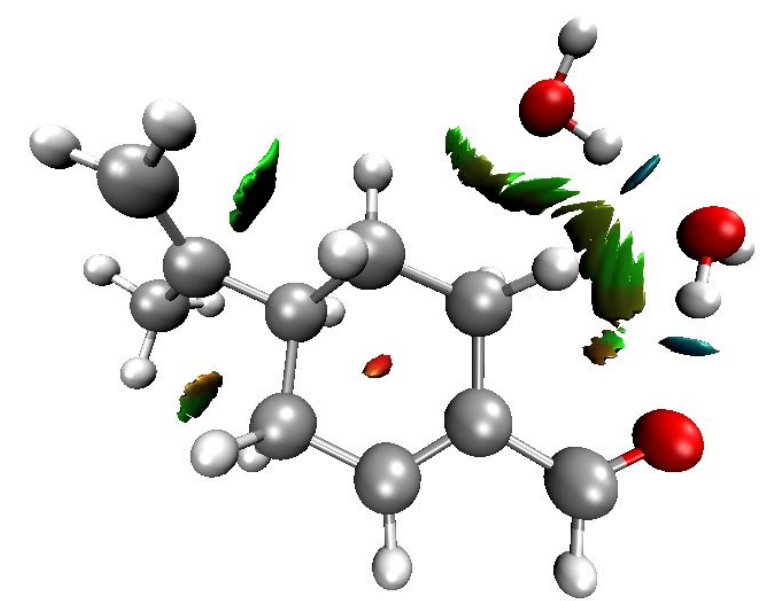

(d) EQ2-2w(II)
Strong attractive interaction (hydrogen bond)
Weak interactions (dispersion, Van der Waals)
Strong repulsive interactions (steric)

Figure S4: NCI plots showing the interactions in the dihydrates of perillaldehyde. The parameters used are $(s=0.5)$ and $\operatorname{sign}\left(\lambda_{2}\right) \rho$ between $-0.05 \mathrm{a} u$ and $+0.05 \mathrm{a} u$. 
Table S6: Calculated rotational constants, dipole moments and relative energies (including ZPE corrections) of the four dihydrates of perillaldehyde.

\begin{tabular}{|c|c|c|c|c|}
\hline Parameter & $\operatorname{DFT}^{a}$ & $\mathrm{MP}^{b}$ & DFT $^{a}$ & $\mathrm{MP} 2^{b}$ \\
\hline & \multicolumn{2}{|c|}{ EQ1-2w(I) } & \multicolumn{2}{|c|}{ EQ1-2w(II) } \\
\hline$A / \mathrm{MHz}$ & 1823.94 & 1781.95 & 983.97 & 968.50 \\
\hline$B / \mathrm{MHz}$ & 253.11 & 252.50 & 394.63 & 402.32 \\
\hline $\mathrm{C} / \mathrm{MHz}$ & 235.11 & 234.09 & 303.46 & 307.04 \\
\hline$\left|\mu_{a}\right| / \mathrm{D}$ & 2.99 & 2.14 & 1.56 & 0.86 \\
\hline$\left|\mu_{b}\right| / \mathrm{D}$ & 0.26 & 0.06 & 2.41 & 2.01 \\
\hline$\left|\mu_{c}\right| / \mathrm{D}$ & 0.54 & 0.48 & 0.19 & 0.01 \\
\hline \multirow[t]{2}{*}{$\Delta E^{c} / \mathrm{kJ} \mathrm{mol}^{-1}$} & 0.0 & 0.0 & 0.85 & 0.98 \\
\hline & \multicolumn{2}{|c|}{ EQ2-2w(I) } & \multicolumn{2}{|c|}{ EQ2-2w(II) } \\
\hline$A / \mathrm{MHz}$ & 1802.07 & 1752.47 & 1059.78 & 985.96 \\
\hline$B / \mathrm{MHz}$ & 255.47 & 255.33 & 369.29 & 398.32 \\
\hline $\mathrm{C} / \mathrm{MHz}$ & 231.21 & 230.59 & 311.34 & 300.85 \\
\hline$\left|\mu_{a}\right| / \mathrm{D}$ & 3.01 & 2.12 & 2.01 & 1.03 \\
\hline$\left|\mu_{b}\right| / \mathrm{D}$ & 0.54 & 0.31 & 1.92 & 2.18 \\
\hline$\left|\mu_{c}\right| / \mathrm{D}$ & 0.03 & 0.03 & 0.15 & 0.43 \\
\hline$\Delta E^{c} / \mathrm{kJ} \mathrm{mol}^{-1}$ & 1.34 & 1.35 & 1.99 & 1.99 \\
\hline
\end{tabular}

${ }^{a}$ B3LYP-D3BJ/def2-TZVP $;{ }^{b} \mathrm{MP} 2$ / 6-311++G(d,p) ; ${ }^{c}$ Energy gap relative to conformer EQ1-2w(I) (including ZPE correction). 
Table S7: Experimental rotational and quartic centrifugal distortion constants of the normal and of the $\mathrm{H}_{2}^{18} \mathrm{O}$ substituted species of the EQ1-2w(I) hydrate of perillaldehyde.

\begin{tabular}{lccc}
\hline & Normal & $\mathrm{MP2}^{a}$ & $\mathrm{DFT}^{b}$ \\
\hline $\mathrm{A} / \mathrm{MHz}$ & $1781.847(16)$ & 1781.95 & 1823.94 \\
$\mathrm{~B} / \mathrm{MHz}$ & $251.035817(74)$ & 252.49 & 253.11 \\
$\mathrm{C} / \mathrm{MHz}$ & $232.790458(64)$ & 234.09 & 235.11 \\
$\Delta_{J} / \mathrm{kHz}$ & $0.014131(77)$ & 0.011 & 0.0080 \\
$\Delta_{J \mathrm{~K}} / \mathrm{kHz}$ & $-0.1609(37)$ & -0.161 & -0.0714 \\
$\Delta_{K} / \mathrm{kHz}$ & - & 2.390 & 1.6312 \\
$\delta_{J} / \mathrm{kHz}$ & $0.00234(10)$ & 0.002 & 0.0011 \\
$\delta_{K} / \mathrm{kHz}$ & - & -0.044 & -0.0937 \\
$\sigma / \mathrm{kHz}$ & 1.01 & - & - \\
$N_{\text {lines }}$ & 46 & - & - \\
$\kappa$ & -0.976 & -0.976 & -0.977 \\
\hline & $18 \mathrm{O}_{\mathrm{w} 1^{-1}}{ }^{18} \mathrm{O}_{\mathrm{w} 2}$ & $18 \mathrm{O}_{\mathrm{w} 1^{-1}{ }^{16} \mathrm{O}_{\mathrm{w} 2}} 16 \mathrm{O}_{\mathrm{w} 1^{1}{ }^{18} \mathrm{O}_{\mathrm{w} 2}}$ \\
\hline $\mathrm{A} / \mathrm{MHz}$ & $1726.585(31)$ & $1776.65(16)$ & $1732.539(72)$ \\
$B / \mathrm{MHz}$ & $239.686977(41)$ & $244.17755(12)$ & $246.155028(87)$ \\
$C / \mathrm{MHz}$ & $222.110607(53)$ & $226.80080(10)$ & $227.75575(13)$ \\
$\Delta_{J} / \mathrm{kHz}$ & $0.01348(22)$ & $0.01402(33)$ & $0.01436(47)$ \\
$\Delta_{J K} / \mathrm{kHz}$ & $c$ & $-0.190(16)$ & $-0.186(17)$ \\
$\delta_{J} / \mathrm{kHz}$ & $c$ & $c$ & $c$ \\
$\sigma / \mathrm{kHz}$ & 0.55 & 1.79 & 0.51 \\
$N_{\text {lines }}$ & 13 & 12 & 9 \\
$\kappa$ & -0.977 & -0.977 & -0.976 \\
\hline & $c$ & & \\
\hline
\end{tabular}

${ }^{a} \mathrm{MP} 2 / 6-311++\mathrm{G}(\mathrm{d}, \mathrm{p}){ }^{b}{ }^{b} \mathrm{~B} 3 \mathrm{LYP}-\mathrm{D} 3 \mathrm{BJ} / \mathrm{def2}-\mathrm{TZVP} ;{ }^{c}$ Fixed at the value of the parent species. 
Table S8: Experimental rotational and quartic centrifugal distortion constants of the normal and of the $\mathrm{H}_{2}^{18} \mathrm{O}$ substituted species of the EQ2-2w(I) hydrate of perillaldehyde.

\begin{tabular}{|c|c|c|c|}
\hline & Normal & $\mathrm{MP}^{a}$ & $\mathrm{DFT}^{b}$ \\
\hline $\mathrm{A} / \mathrm{MHz}$ & $1756.6376(71)$ & 1752.47 & 1802.07 \\
\hline B / MHz & $253.482784(30)$ & 255.33 & 255.47 \\
\hline $\mathrm{C} / \mathrm{MHz}$ & $229.299435(28)$ & 230.59 & 231.21 \\
\hline$\Delta_{J} / \mathrm{kHz}$ & $0.014676(48)$ & 0.012 & 0.0086 \\
\hline$\Delta_{J K} / \mathrm{kHz}$ & $-0.2396(28)$ & -0.1823 & -0.1023 \\
\hline$\Delta_{K} / \mathrm{kHz}$ & - & 2.483 & 1.803 \\
\hline$\delta_{J} / \mathrm{kHz}$ & $0.002636(55)$ & 0.0021 & 0.0014 \\
\hline$\delta_{K} / \mathrm{kHz}$ & - & 0.077 & 0.0648 \\
\hline$\sigma / \mathrm{kHz}$ & 0.50 & - & - \\
\hline$N_{\text {lines }}$ & 36 & - & - \\
\hline \multirow[t]{2}{*}{$\kappa$} & -0.968 & -0.967 & -0.969 \\
\hline & ${ }^{18} \mathrm{O}_{\mathrm{w} 1}{ }^{18} \mathrm{O}_{\mathrm{w} 2}$ & ${ }^{18} \mathrm{O}_{\mathrm{w} 1}{ }^{16} \mathrm{O}_{\mathrm{w} 2}$ & ${ }^{16} \mathrm{O}_{\mathrm{w} 1}{ }^{18} \mathrm{O}_{\mathrm{w} 2}$ \\
\hline $\mathrm{A} / \mathrm{MHz}$ & 1702.22(10) & $1752.206(41)$ & $1707.203(14)$ \\
\hline$B / \mathrm{MHz}$ & $241.91204(18)$ & $246.45711(22)$ & $248.539100(89)$ \\
\hline $\mathrm{C} / \mathrm{MHz}$ & 218.95332(31) & 223.47723(13) & $224.445820(69)$ \\
\hline$\Delta_{J} / \mathrm{kHz}$ & $0.01412(26)$ & $0.01388(38)$ & $0.01401(11)$ \\
\hline$\Delta_{J K} / \mathrm{kHz}$ & $-0.254(15)$ & $-0.249(23)$ & $-0.2150(37)$ \\
\hline$\delta_{J} / \mathrm{kHz}$ & $0.00347(49)$ & $0.00244(39)$ & $0.00204(23)$ \\
\hline$\sigma / \mathrm{kHz}$ & 0.48 & 1.51 & 0.12 \\
\hline$N_{\text {lines }}$ & 11 & 11 & 10 \\
\hline$\kappa$ & -0.969 & -0.970 & -0.967 \\
\hline
\end{tabular}

${ }^{a} \mathrm{MP2} /$ 6-311++G(d,p); ${ }^{b}$ B3LYP-D3BJ/def2-TZVP; 


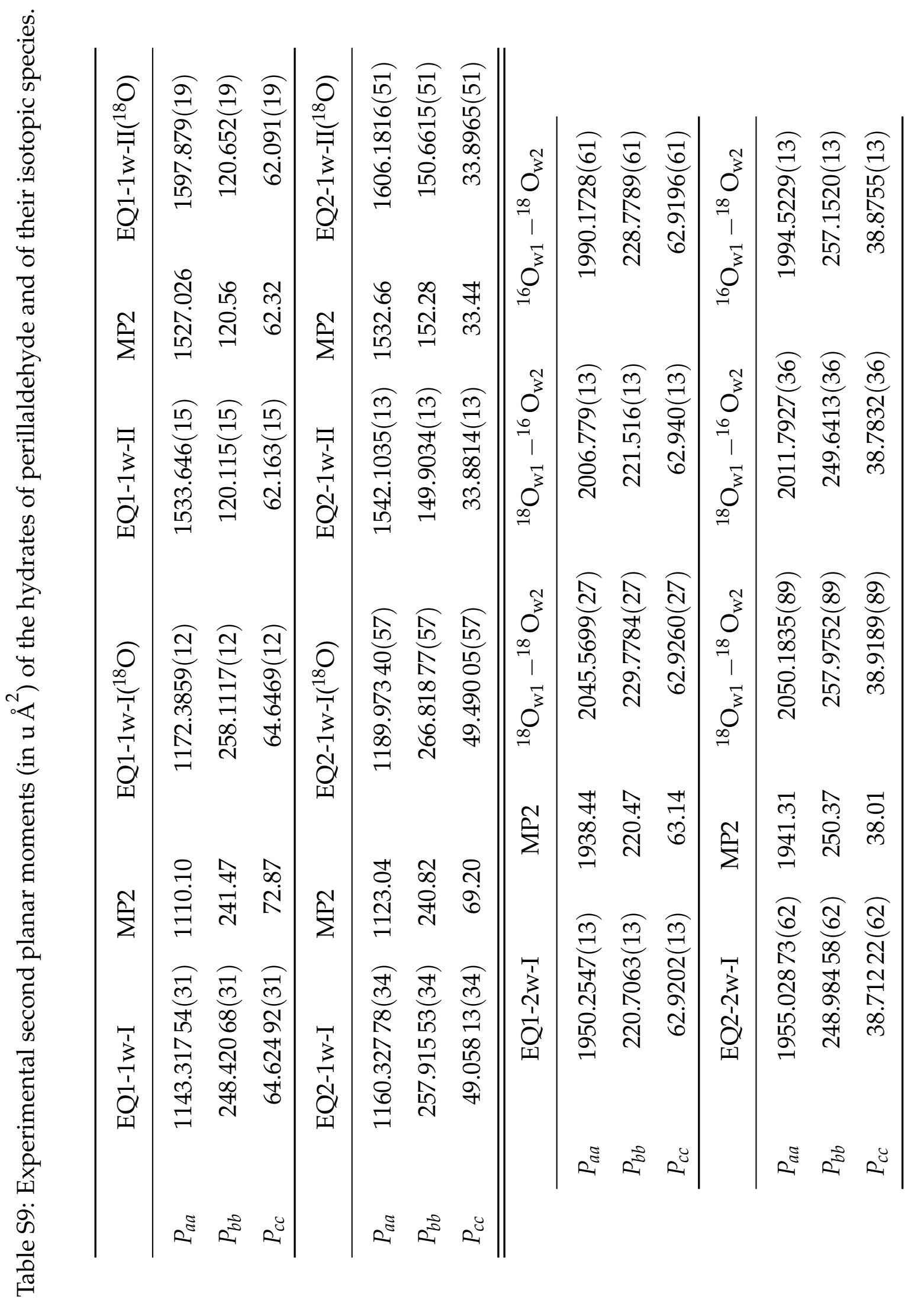




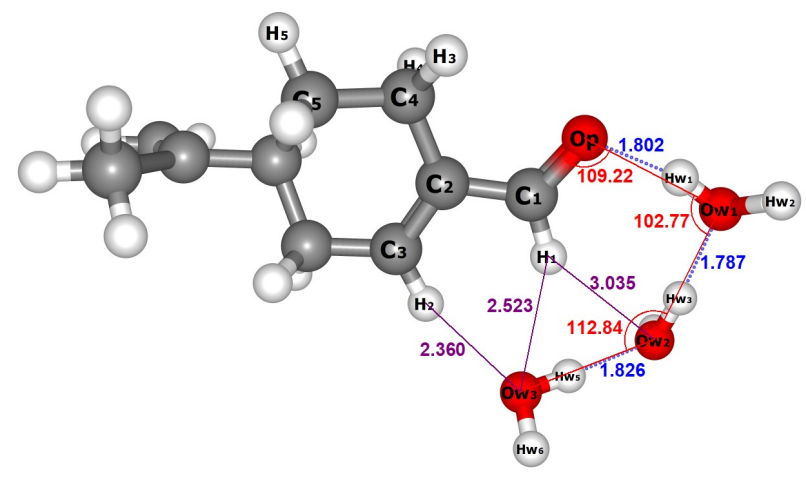

(a) EQ1-3w

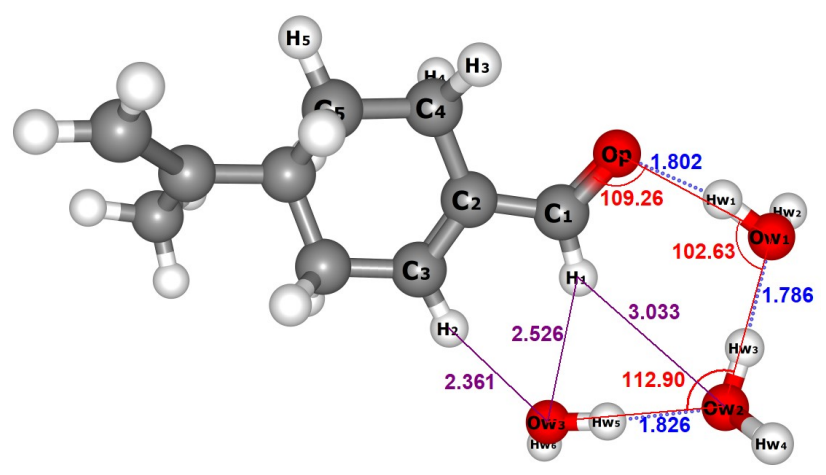

(b) EQ2-3w $\left(2.13 \mathrm{~kJ} \mathrm{~mol}^{-1}\right)$

Figure S5: The structures of the trihydrates 3w(I) with the two parent molecules of perillaldehyde. The values in blue are relative to the hydrogen bond. The shortest distances between oxygen atoms of water and hydrogen atoms of perillaldehyde are indicated in purple, and the angles are shown in red. Lengths are expressed in $\AA$ and angles in ${ }^{\circ}$. 
Table S10: Measured frequencies $\left(v_{\mathrm{obs}}\right)$ and residuals $\left(v_{\mathrm{obs}}-v_{\text {calc. }}\right)$ in $\mathrm{MHz}$ of the monohydrate of perillaldehyde EQ1-1w(I).

\begin{tabular}{rrrrrrrrrr}
\hline$J^{\prime}$ & $K_{a}^{\prime}$ & $K_{c}^{\prime}$ & $\leftarrow$ & $J^{\prime \prime}$ & $K_{a}^{\prime \prime}$ & $K_{c}^{\prime \prime}$ & $v_{\text {obs. }}$ & $v_{\text {obs. }}-v_{\text {calc. }}$ & exp. error \\
\hline 4 & 1 & 4 & $\leftarrow$ & 3 & 1 & 3 & 3011.8881 & -0.0008 & 0.002 \\
4 & 1 & 4 & $\leftarrow$ & 3 & 1 & 3 & 3011.8881 & -0.0008 & 0.002 \\
4 & 0 & 4 & $\leftarrow$ & 3 & 0 & 3 & 3107.4435 & -0.0006 & 0.002 \\
4 & 1 & 3 & $\leftarrow$ & 3 & 1 & 2 & 3232.6634 & 0.0000 & 0.002 \\
5 & 1 & 5 & $\leftarrow$ & 4 & 1 & 4 & 3761.6073 & -0.0006 & 0.002 \\
5 & 0 & 5 & $\leftarrow$ & 4 & 0 & 4 & 3870.7408 & -0.0005 & 0.002 \\
5 & 2 & 3 & $\leftarrow$ & 4 & 2 & 2 & 3940.1304 & 0.0009 & 0.002 \\
5 & 1 & 4 & $\leftarrow$ & 4 & 1 & 3 & 4037.0416 & 0.0000 & 0.002 \\
6 & 1 & 6 & $\leftarrow$ & 5 & 1 & 5 & 4509.363 & -0.0012 & 0.002 \\
6 & 0 & 6 & $\leftarrow$ & 5 & 0 & 5 & 4625.79 & 0.0001 & 0.002 \\
7 & 0 & 7 & $\leftarrow$ & 6 & 1 & 6 & 4627.1726 & 0.0008 & 0.002 \\
6 & 2 & 5 & $\leftarrow$ & 5 & 2 & 4 & 4680.5915 & -0.0009 & 0.002 \\
6 & 2 & 4 & $\leftarrow$ & 5 & 2 & 3 & 4743.7924 & 0.0004 & 0.002 \\
6 & 1 & 5 & $\leftarrow$ & 5 & 1 & 4 & 4838.6701 & -0.0013 & 0.002 \\
7 & 1 & 7 & $\leftarrow$ & 6 & 1 & 6 & 5254.9589 & -0.0007 & 0.002 \\
7 & 0 & 7 & $\leftarrow$ & 6 & 0 & 6 & 5372.1243 & -0.0003 & 0.002 \\
7 & 2 & 6 & $\leftarrow$ & 6 & 2 & 5 & 5455.9674 & -0.0005 & 0.002 \\
8 & 0 & 8 & $\leftarrow$ & 7 & 1 & 7 & 5482.367 & 0.0007 & 0.002 \\
7 & 2 & 5 & $\leftarrow$ & 6 & 2 & 4 & 5554.2629 & 0.0000 & 0.002 \\
7 & 1 & 6 & $\leftarrow$ & 6 & 1 & 5 & 5636.7809 & 0.0003 & 0.002 \\
8 & 1 & 8 & $\leftarrow$ & 7 & 1 & 7 & 5998.2878 & 0.0023 & 0.002 \\
8 & 0 & 8 & $\leftarrow$ & 7 & 0 & 7 & 6110.1552 & 0.0010 & 0.002 \\
8 & 2 & 7 & $\leftarrow$ & 7 & 2 & 6 & 6229.1889 & -0.0009 & 0.002 \\
8 & 3 & 6 & $\leftarrow$ & 7 & 3 & 5 & 6269.6717 & 0.0041 & 0.002 \\
9 & 0 & 9 & $\leftarrow$ & 8 & 1 & 8 & 6325.2149 & -0.0004 & 0.002 \\
8 & 2 & 6 & $\leftarrow$ & 7 & 2 & 5 & 6370.7919 & 0.0000 & 0.002 \\
8 & 1 & 7 & $\leftarrow$ & 7 & 1 & 6 & 6430.4824 & -0.0001 & 0.001 \\
8 & 1 & 8 & $\leftarrow$ & 7 & 0 & 7 & 6626.0739 & 0.0005 & 0.002 \\
9 & 1 & 9 & $\leftarrow$ & 8 & 1 & 8 & 6739.3226 & 0.0008 & 0.002 \\
9 & 0 & 9 & $\leftarrow$ & 8 & 0 & 8 & 6841.1347 & 0.0002 & 0.002 \\
\hline & & & & & & & & &
\end{tabular}




\begin{tabular}{rrrrrrrrrr}
\hline$J^{\prime}$ & $K_{a}^{\prime}$ & $K_{c}^{\prime}$ & $\leftarrow$ & $J^{\prime \prime}$ & $K^{\prime \prime}{ }_{a}$ & $K^{\prime \prime}{ }_{c}$ & $v_{\text {obs. }}$ & $v_{\text {obs. }}-v_{\text {calc. }}$ & exp. error \\
\hline 9 & 2 & 8 & $\leftarrow$ & 8 & 2 & 7 & 6999.981 & -0.0002 & 0.002 \\
9 & 3 & 7 & $\leftarrow$ & 8 & 3 & 6 & 7056.111 & 0.0012 & 0.002 \\
9 & 3 & 6 & $\leftarrow$ & 8 & 3 & 5 & 7073.9032 & -0.0024 & 0.002 \\
10 & 0 & 10 & $\leftarrow$ & 9 & 1 & 9 & 7152.7746 & -0.0024 & 0.002 \\
9 & 2 & 7 & $\leftarrow$ & 8 & 2 & 6 & 7191.7595 & 0.0000 & 0.002 \\
9 & 1 & 8 & $\leftarrow$ & 8 & 1 & 7 & 7218.7769 & 0.0005 & 0.002 \\
9 & 1 & 9 & $\leftarrow$ & 8 & 0 & 8 & 7255.2395 & -0.0013 & 0.002 \\
10 & 1 & 10 & $\leftarrow$ & 9 & 1 & 9 & 7478.1308 & -0.0002 & 0.002 \\
10 & 0 & 10 & $\leftarrow$ & 9 & 0 & 9 & 7566.885 & 0.0015 & 0.002 \\
10 & 2 & 9 & $\leftarrow$ & 9 & 2 & 8 & 7768.0878 & -0.0001 & 0.002 \\
10 & 4 & 7 & $\leftarrow$ & 9 & 4 & 6 & 7838.272 & -0.0004 & 0.002 \\
10 & 4 & 6 & $\leftarrow$ & 9 & 4 & 5 & 7839.4508 & -0.0008 & 0.002 \\
10 & 3 & 8 & $\leftarrow$ & 9 & 3 & 7 & 7842.6563 & 0.0003 & 0.002 \\
10 & 1 & 10 & $\leftarrow$ & 9 & 0 & 9 & 7892.2372 & -0.0002 & 0.002 \\
10 & 1 & 9 & $\leftarrow$ & 9 & 1 & 8 & 8000.572 & 0.0004 & 0.002 \\
10 & 2 & 8 & $\leftarrow$ & 9 & 2 & 7 & 8014.951 & 0.0003 & 0.002 \\
\hline
\end{tabular}

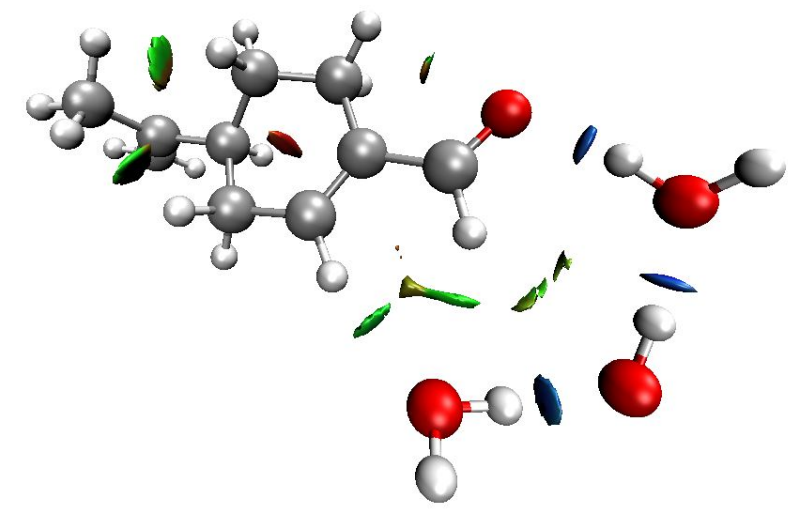

(a) EQ1-3w

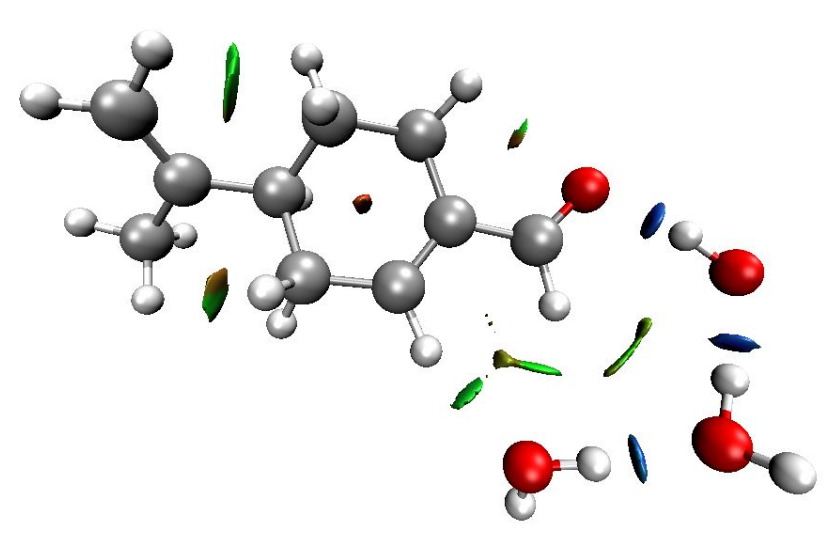

(b) EQ2-3w
Strong attractive interaction

(hydrogen bond)
Weak interactions (dispersion, Van der Waals)
Strong repulsive interactions (steric)

Figure S6: NCI plots showing the interactions in the trihydrates of perillaldehyde. The parameters used are $(s=0.5)$ and $\operatorname{sign}\left(\lambda_{2}\right) \rho$ between $-0.05 \mathrm{a} u$ and $+0.05 \mathrm{a} \mathrm{u}$. 
Table S11: Measured frequencies ( $\left.v_{\text {obs. }}\right)$ and residuals $\left(v_{\text {obs. }}-v_{\text {calc. }}\right)$ in $\mathrm{MHz}$ of the monohydrate of perillaldehyde EQ1-1w(I) labeled with ${ }^{18} \mathrm{O}$.

\begin{tabular}{|c|c|c|c|c|c|c|c|c|c|}
\hline$J^{\prime}$ & $K_{a}^{\prime}$ & $K_{c}^{\prime}$ & $\leftarrow$ & $J^{\prime \prime}$ & $K^{\prime \prime} a$ & $K^{\prime \prime}{ }_{c}$ & $v_{\text {obs. }}$ & $v_{\text {obs. }}-v_{\text {calc }}$ & exp. error \\
\hline 4 & 0 & 4 & $\leftarrow$ & 3 & 0 & 3 & 3028.1339 & -0.0004 & 0.001 \\
\hline 5 & 1 & 5 & $\leftarrow$ & 4 & 1 & 4 & 3662.9775 & 0.0000 & 0.002 \\
\hline 5 & 0 & 5 & $\leftarrow$ & 4 & 0 & 4 & 3771.1916 & -0.0005 & 0.001 \\
\hline 5 & 1 & 4 & $\leftarrow$ & 4 & 1 & 3 & 3938.3561 & -0.0002 & 0.002 \\
\hline 6 & 1 & 6 & $\leftarrow$ & 5 & 1 & 5 & 4390.8783 & 0.0006 & 0.002 \\
\hline 6 & 0 & 6 & $\leftarrow$ & 5 & 0 & 5 & 4505.8016 & 0.0004 & 0.001 \\
\hline 6 & 2 & 5 & $\leftarrow$ & 5 & 2 & 4 & 4562.2516 & 0.0011 & 0.002 \\
\hline 6 & 2 & 4 & $\leftarrow$ & 5 & 2 & 3 & 4627.3679 & -0.0002 & 0.001 \\
\hline 6 & 1 & 5 & $\leftarrow$ & 5 & 1 & 4 & 4720.0389 & 0.0002 & 0.001 \\
\hline 7 & 1 & 7 & $\leftarrow$ & 6 & 1 & 6 & 5116.5645 & -0.0020 & 0.002 \\
\hline 7 & 0 & 7 & $\leftarrow$ & 6 & 0 & 6 & 5231.5567 & -0.0001 & 0.002 \\
\hline 7 & 2 & 6 & $\leftarrow$ & 6 & 2 & 5 & 5317.7493 & 0.0001 & 0.002 \\
\hline 7 & 1 & 6 & $\leftarrow$ & 6 & 1 & 5 & 5498.0618 & 0.0005 & 0.002 \\
\hline 8 & 1 & 8 & $\leftarrow$ & 7 & 1 & 7 & 5839.9419 & 0.0003 & 0.002 \\
\hline 8 & 0 & 8 & $\leftarrow$ & 7 & 0 & 7 & 5948.9785 & -0.0002 & 0.002 \\
\hline 8 & 2 & 7 & $\leftarrow$ & 7 & 2 & 6 & 6071.0259 & -0.0003 & 0.002 \\
\hline 8 & 1 & 7 & $\leftarrow$ & 7 & 1 & 6 & 6271.4972 & 0.0010 & 0.002 \\
\hline 9 & 1 & 9 & $\leftarrow$ & 8 & 1 & 8 & 6560.996 & 0.0028 & 0.002 \\
\hline 9 & 0 & 9 & $\leftarrow$ & 8 & 0 & 8 & 6659.4551 & 0.0018 & 0.002 \\
\hline 9 & 2 & 8 & $\leftarrow$ & 8 & 2 & 7 & 6821.798 & 0.0000 & 0.002 \\
\hline 9 & 1 & 8 & $\leftarrow$ & 8 & 1 & 7 & 7039.2956 & -0.0010 & 0.002 \\
\hline 10 & 1 & 10 & $\leftarrow$ & 9 & 1 & 9 & 7279.796 & -0.0007 & 0.002 \\
\hline 10 & 0 & 10 & $\leftarrow$ & 9 & 0 & 9 & 7364.8998 & -0.0019 & 0.002 \\
\hline
\end{tabular}


Table S12: Measured frequencies $\left(v_{\mathrm{obs}}\right)$ and residuals $\left(v_{\mathrm{obs}}-v_{\text {calc. }}\right)$ in $\mathrm{MHz}$ of the monohydrate of perillaldehyde EQ1-1w(II).

\begin{tabular}{rrrrrrrrrr}
\hline$J^{\prime}$ & $K_{a}^{\prime}$ & $K_{c}^{\prime}$ & $\leftarrow$ & $J^{\prime \prime}$ & $K_{a}^{\prime \prime}$ & $K^{\prime \prime}{ }_{c}$ & $v_{\text {obs. }}$ & $v_{\text {obs. }}-v_{\text {calc. }}$ & exp. error \\
\hline 4 & 0 & 4 & $\leftarrow$ & 3 & 0 & 3 & 2488.7606 & 0.0000 & 0.002 \\
4 & 1 & 3 & $\leftarrow$ & 3 & 1 & 2 & 2511.2594 & 0.0021 & 0.002 \\
5 & 1 & 5 & $\leftarrow$ & 4 & 1 & 4 & 3083.5107 & -0.0004 & 0.002 \\
5 & 0 & 5 & $\leftarrow$ & 4 & 0 & 4 & 3110.6665 & 0.0000 & 0.002 \\
5 & 1 & 4 & $\leftarrow$ & 4 & 1 & 3 & 3138.9997 & 0.0018 & 0.002 \\
6 & 1 & 6 & $\leftarrow$ & 5 & 1 & 5 & 3700.1054 & -0.0018 & 0.002 \\
6 & 0 & 6 & $\leftarrow$ & 5 & 0 & 5 & 3732.3836 & 0.0003 & 0.002 \\
6 & 1 & 5 & $\leftarrow$ & 5 & 1 & 4 & 3766.6908 & 0.0019 & 0.002 \\
7 & 1 & 7 & $\leftarrow$ & 6 & 1 & 6 & 4316.6451 & -0.0010 & 0.001 \\
7 & 0 & 7 & $\leftarrow$ & 6 & 0 & 6 & 4353.8726 & -0.0005 & 0.002 \\
7 & 2 & 6 & $\leftarrow$ & 6 & 2 & 5 & 4355.6936 & 0.0025 & 0.002 \\
7 & 2 & 5 & $\leftarrow$ & 6 & 2 & 4 & 4357.7886 & -0.0024 & 0.002 \\
7 & 1 & 6 & $\leftarrow$ & 6 & 1 & 5 & 4394.3219 & 0.0019 & 0.002 \\
8 & 1 & 8 & $\leftarrow$ & 7 & 1 & 7 & 4933.118 & -0.0009 & 0.002 \\
8 & 0 & 8 & $\leftarrow$ & 7 & 0 & 7 & 4975.0996 & 0.0006 & 0.002 \\
8 & 2 & 7 & $\leftarrow$ & 7 & 2 & 6 & 4977.7959 & -0.0040 & 0.002 \\
8 & 1 & 7 & $\leftarrow$ & 7 & 1 & 6 & 5021.8815 & 0.0010 & 0.002 \\
9 & 1 & 9 & $\leftarrow$ & 8 & 1 & 8 & 5549.5175 & 0.0004 & 0.002 \\
9 & 0 & 9 & $\leftarrow$ & 8 & 0 & 8 & 5596.0237 & -0.0002 & 0.002 \\
9 & 1 & 8 & $\leftarrow$ & 8 & 1 & 7 & 5649.3601 & 0.0005 & 0.002 \\
10 & 1 & 10 & $\leftarrow$ & 9 & 1 & 9 & 6165.8326 & 0.0006 & 0.001 \\
10 & 0 & 10 & $\leftarrow$ & 9 & 0 & 9 & 6216.6127 & 0.0005 & 0.002 \\
10 & 2 & 9 & $\leftarrow$ & 9 & 2 & 8 & 6221.8536 & 0.0014 & 0.002 \\
10 & 2 & 8 & $\leftarrow$ & 9 & 2 & 7 & 6228.0259 & -0.0006 & 0.002 \\
10 & 1 & 9 & $\leftarrow$ & 9 & 1 & 8 & 6276.7455 & -0.0005 & 0.001 \\
11 & 1 & 11 & $\leftarrow$ & 10 & 1 & 10 & 6782.0581 & 0.0023 & 0.002 \\
11 & 0 & 11 & $\leftarrow$ & 10 & 0 & 10 & 6836.8289 & 0.0001 & 0.002 \\
11 & 2 & 10 & $\leftarrow$ & 10 & 2 & 9 & 6843.7841 & 0.0019 & 0.002 \\
11 & 1 & 10 & $\leftarrow$ & 10 & 1 & 9 & 6904.0257 & -0.0026 & 0.002 \\
\hline 2 & 0 & 12 & $\leftarrow$ & 11 & 0 & 11 & 7456.6386 & -0.0014 & 0.002 \\
\hline & & & & & & & & &
\end{tabular}


Table S13: Measured frequencies $\left(v_{\text {obs. }}\right)$ and residuals $\left(v_{\text {obs. }}-v_{\text {calc. }}\right)$ in $\mathrm{MHz}$ of the monohydrate of perillaldehyde EQ1-1w(II) labeled with ${ }^{18} \mathrm{O}$.

\begin{tabular}{rrrrrrrrrr}
\hline$J^{\prime}$ & $K_{a}^{\prime}$ & $K_{c}^{\prime}$ & $\leftarrow$ & $J^{\prime \prime}$ & $K^{\prime \prime}{ }_{a}$ & $K^{\prime \prime}{ }_{c}$ & $v_{\text {obs. }}$ & $v_{\text {obs. }}-v_{\text {calc. }}$ & exp. error \\
\hline 5 & 0 & 5 & $\leftarrow$ & 4 & 0 & 4 & 2991.9774 & 0.0006 & 0.002 \\
5 & 1 & 4 & $\leftarrow$ & 4 & 1 & 3 & 3018.4274 & 0.0010 & 0.002 \\
6 & 1 & 6 & $\leftarrow$ & 5 & 1 & 5 & 3559.7709 & -0.0018 & 0.002 \\
6 & 0 & 6 & $\leftarrow$ & 5 & 0 & 5 & 3590.0074 & -0.0002 & 0.002 \\
6 & 1 & 5 & $\leftarrow$ & 5 & 1 & 4 & 3622.0167 & 0.0006 & 0.002 \\
7 & 1 & 7 & $\leftarrow$ & 6 & 1 & 6 & 4152.9386 & -0.0011 & 0.002 \\
7 & 0 & 7 & $\leftarrow$ & 6 & 0 & 6 & 4187.8393 & -0.0008 & 0.002 \\
7 & 1 & 6 & $\leftarrow$ & 6 & 1 & 5 & 4225.5536 & 0.0005 & 0.002 \\
8 & 1 & 8 & $\leftarrow$ & 7 & 1 & 7 & 4746.0483 & 0.0000 & 0.002 \\
8 & 0 & 8 & $\leftarrow$ & 7 & 0 & 7 & 4785.4413 & -0.0003 & 0.002 \\
8 & 1 & 7 & $\leftarrow$ & 7 & 1 & 6 & 4829.0283 & 0.0003 & 0.002 \\
10 & 1 & 10 & $\leftarrow$ & 9 & 1 & 9 & 5932.0619 & 0.0022 & 0.002 \\
10 & 0 & 10 & $\leftarrow$ & 9 & 0 & 9 & 5979.8222 & -0.0006 & 0.002 \\
10 & 1 & 9 & $\leftarrow$ & 9 & 1 & 8 & 6035.7534 & 0.0000 & 0.002 \\
11 & 0 & 11 & $\leftarrow$ & 10 & 0 & 10 & 6576.5409 & 0.0008 & 0.002 \\
12 & 0 & 12 & $\leftarrow$ & 11 & 0 & 11 & 7172.9015 & 0.0000 & 0.002 \\
12 & 2 & 11 & $\leftarrow$ & 11 & 2 & 10 & 7180.77 & -0.0001 & 0.002 \\
12 & 1 & 11 & $\leftarrow$ & 11 & 1 & 10 & 7242.1119 & -0.0011 & 0.002 \\
\hline & & & & & & & & & \\
\hline
\end{tabular}


Table S14: Measured frequencies $\left(v_{\mathrm{obs}}\right)$ and residuals $\left(v_{\mathrm{obs}}-v_{\text {calc. }}\right)$ in $\mathrm{MHz}$ of the monohydrate of perillaldehyde EQ2-1w(I).

\begin{tabular}{rrrrrrrrrr}
\hline$J^{\prime}$ & $K_{a}^{\prime}$ & $K_{c}^{\prime}$ & $\leftarrow$ & $J^{\prime \prime}$ & $K^{\prime \prime}{ }_{a}$ & $K_{c}^{\prime \prime}$ & \multicolumn{1}{c}{$v_{\text {obs. }}$} & $v_{\text {obs. }}-v_{\text {calc. }}$ & exp. error \\
\hline 3 & 0 & 3 & $\leftarrow$ & 2 & 0 & 2 & 2313.665 & 0.0007 & 0.002 \\
4 & 1 & 4 & $\leftarrow$ & 3 & 1 & 3 & 2969.4432 & 0.0003 & 0.002 \\
4 & 0 & 4 & $\leftarrow$ & 3 & 0 & 3 & 3074.5144 & 0.0009 & 0.001 \\
5 & 1 & 5 & $\leftarrow$ & 4 & 1 & 4 & 3707.908 & 0.0001 & 0.002 \\
5 & 0 & 5 & $\leftarrow$ & 4 & 0 & 4 & 3826.8884 & 0.0000 & 0.002 \\
5 & 2 & 4 & $\leftarrow$ & 4 & 2 & 3 & 3866.0777 & 0.0022 & 0.002 \\
5 & 1 & 4 & $\leftarrow$ & 4 & 1 & 3 & 4014.5173 & 0.0000 & 0.001 \\
6 & 1 & 6 & $\leftarrow$ & 5 & 1 & 5 & 4444.0427 & -0.0003 & 0.001 \\
6 & 0 & 6 & $\leftarrow$ & 5 & 0 & 5 & 4569.5288 & 0.0002 & 0.001 \\
6 & 2 & 5 & $\leftarrow$ & 5 & 2 & 4 & 4635.1556 & -0.0002 & 0.002 \\
6 & 2 & 4 & $\leftarrow$ & 5 & 2 & 3 & 4710.8857 & -0.0011 & 0.002 \\
6 & 1 & 5 & $\leftarrow$ & 5 & 1 & 4 & 4810.3981 & 0.0000 & 0.002 \\
7 & 1 & 7 & $\leftarrow$ & 6 & 1 & 6 & 5177.6325 & 0.0000 & 0.002 \\
7 & 0 & 7 & $\leftarrow$ & 6 & 0 & 6 & 5302.0955 & 0.0003 & 0.002 \\
6 & 1 & 6 & $\leftarrow$ & 5 & 0 & 5 & 5304.4562 & -0.0040 & 0.002 \\
7 & 2 & 6 & $\leftarrow$ & 6 & 2 & 5 & 5401.9974 & -0.0008 & 0.002 \\
8 & 0 & 8 & $\leftarrow$ & 7 & 1 & 7 & 5414.891 & -0.0014 & 0.002 \\
7 & 3 & 5 & $\leftarrow$ & 6 & 3 & 4 & 5435.1008 & -0.0004 & 0.002 \\
7 & 3 & 4 & $\leftarrow$ & 6 & 3 & 3 & 5441.5345 & -0.0009 & 0.002 \\
7 & 2 & 5 & $\leftarrow$ & 6 & 2 & 4 & 5519.2784 & -0.0016 & 0.002 \\
7 & 1 & 6 & $\leftarrow$ & 6 & 1 & 5 & 5601.9679 & 0.0000 & 0.002 \\
8 & 1 & 8 & $\leftarrow$ & 7 & 1 & 7 & 5908.5737 & 0.0004 & 0.002 \\
8 & 0 & 8 & $\leftarrow$ & 7 & 0 & 7 & 6025.3627 & 0.0011 & 0.002 \\
8 & 2 & 7 & $\leftarrow$ & 7 & 2 & 6 & 6166.2532 & -0.0008 & 0.002 \\
8 & 3 & 5 & $\leftarrow$ & 7 & 3 & 4 & 6227.347 & 0.0039 & 0.002 \\
9 & 0 & 9 & $\leftarrow$ & 8 & 1 & 8 & 6247.4065 & -0.0017 & 0.002 \\
8 & 2 & 6 & $\leftarrow$ & 7 & 2 & 5 & 6334.2562 & -0.0007 & 0.002 \\
8 & 1 & 7 & $\leftarrow$ & 7 & 1 & 6 & 6388.1228 & 0.0006 & 0.002 \\
9 & 1 & 9 & $\leftarrow$ & 8 & 1 & 8 & 6636.8725 & 0.0003 & 0.002 \\
\hline
\end{tabular}




\begin{tabular}{rrrrrrrrrr}
\hline$J^{\prime}$ & $K_{a}^{\prime}$ & $K_{c}^{\prime}$ & $\leftarrow$ & $J^{\prime \prime}$ & $K^{\prime \prime}{ }_{a}$ & $K_{c}^{\prime \prime}{ }_{c}$ & $v_{\text {obs. }}$ & $v_{\text {obs. }}-v_{\text {calc. }}$ & exp. error \\
\hline 9 & 0 & 9 & $\leftarrow$ & 8 & 0 & 8 & 6741.09 & 0.0009 & 0.002 \\
9 & 2 & 8 & $\leftarrow$ & 8 & 2 & 7 & 6927.5966 & -0.0002 & 0.002 \\
10 & 0 & 10 & $\leftarrow$ & 9 & 1 & 9 & 7062.111 & -0.0015 & 0.002 \\
9 & 1 & 9 & $\leftarrow$ & 8 & 0 & 8 & 7130.5524 & -0.0005 & 0.002 \\
9 & 2 & 7 & $\leftarrow$ & 8 & 2 & 6 & 7153.6076 & 0.0000 & 0.002 \\
9 & 1 & 8 & $\leftarrow$ & 8 & 1 & 7 & 7167.6188 & 0.0013 & 0.002 \\
10 & 1 & 10 & $\leftarrow$ & 9 & 1 & 9 & 7362.6357 & 0.0008 & 0.002 \\
10 & 0 & 10 & $\leftarrow$ & 9 & 0 & 9 & 7451.5774 & 0.0010 & 0.002 \\
10 & 1 & 10 & $\leftarrow$ & 9 & 0 & 9 & 7752.0994 & 0.0006 & 0.002 \\
10 & 3 & 8 & $\leftarrow$ & 9 & 3 & 7 & 7774.269 & 0.0003 & 0.002 \\
10 & 3 & 7 & $\leftarrow$ & 9 & 3 & 6 & 7813.1437 & -0.0030 & 0.002 \\
11 & 0 & 11 & $\leftarrow$ & 10 & 1 & 10 & 7858.5662 & -0.0002 & 0.002 \\
10 & 2 & 8 & $\leftarrow$ & 9 & 2 & 7 & 7974.5394 & 0.0005 & 0.001 \\
\hline
\end{tabular}


Table S15: Measured frequencies ( $\left.v_{\text {obs. }}\right)$ and residuals $\left(v_{\text {obs. }}-v_{\text {calc. }}\right)$ in $\mathrm{MHz}$ of the monohydrate of perillaldehyde EQ2-1w(I) labeled with ${ }^{18} \mathrm{O}$.

\begin{tabular}{rrrlrrrrrr}
\hline$J^{\prime}$ & $K_{a}^{\prime}$ & $K_{c}^{\prime}$ & $\leftarrow$ & $J^{\prime \prime}$ & $K_{a}^{\prime \prime}$ & $K^{\prime \prime}{ }_{c}$ & $v_{\text {obs. }}$ & $v_{\text {obs. }}-v_{\text {calc. }}$ & exp. error \\
\hline 5 & 1 & 5 & $\leftarrow$ & 4 & 1 & 4 & 3611.7683 & -0.0021 & 0.002 \\
5 & 0 & 5 & $\leftarrow$ & 4 & 0 & 4 & 3728.7168 & 0.0007 & 0.002 \\
6 & 1 & 6 & $\leftarrow$ & 5 & 1 & 5 & 4328.649 & 0.0008 & 0.002 \\
6 & 0 & 6 & $\leftarrow$ & 5 & 0 & 5 & 4451.6141 & -0.0006 & 0.002 \\
6 & 2 & 5 & $\leftarrow$ & 5 & 2 & 4 & 4517.6533 & 0.0000 & 0.002 \\
6 & 1 & 5 & $\leftarrow$ & 5 & 1 & 4 & 4690.656 & 0.0002 & 0.002 \\
6 & 1 & 5 & $\leftarrow$ & 5 & 1 & 4 & 4690.6562 & 0.0004 & 0.002 \\
7 & 1 & 7 & $\leftarrow$ & 6 & 1 & 6 & 5042.9736 & -0.0011 & 0.002 \\
7 & 0 & 7 & $\leftarrow$ & 6 & 0 & 6 & 5164.4749 & 0.0016 & 0.002 \\
7 & 2 & 6 & $\leftarrow$ & 6 & 2 & 5 & 5264.8668 & 0.0003 & 0.001 \\
7 & 1 & 6 & $\leftarrow$ & 6 & 1 & 5 & 5462.1538 & -0.0001 & 0.001 \\
8 & 1 & 8 & $\leftarrow$ & 7 & 1 & 7 & 5754.6532 & -0.0003 & 0.002 \\
8 & 0 & 8 & $\leftarrow$ & 7 & 0 & 7 & 5868.1427 & 0.0007 & 0.001 \\
8 & 2 & 7 & $\leftarrow$ & 7 & 2 & 6 & 6009.474 & 0.0000 & 0.002 \\
8 & 2 & 6 & $\leftarrow$ & 7 & 2 & 5 & 6178.1168 & 0.0000 & 0.001 \\
8 & 1 & 7 & $\leftarrow$ & 7 & 1 & 6 & 6228.164 & -0.0007 & 0.002 \\
9 & 1 & 9 & $\leftarrow$ & 8 & 1 & 8 & 6463.6993 & 0.0000 & 0.002 \\
9 & 0 & 9 & $\leftarrow$ & 8 & 0 & 8 & 6564.4515 & -0.0024 & 0.002 \\
9 & 2 & 8 & $\leftarrow$ & 8 & 2 & 7 & 6751.1477 & -0.0007 & 0.002 \\
9 & 1 & 8 & $\leftarrow$ & 8 & 1 & 7 & 6987.4261 & 0.0000 & 0.002 \\
10 & 1 & 10 & $\leftarrow$ & 9 & 1 & 9 & 7170.2262 & 0.0000 & 0.002 \\
10 & 0 & 10 & $\leftarrow$ & 9 & 0 & 9 & 7255.743 & -0.0007 & 0.002 \\
\hline 1 & 1 & 11 & $\leftarrow$ & 10 & 1 & 10 & 7874.4283 & 0.0011 & 0.002 \\
\hline & & & & & & & & & \\
\hline
\end{tabular}


Table S16: Measured frequencies $\left(v_{\mathrm{obs}}\right)$ and residuals $\left(v_{\mathrm{obs}}-v_{\text {calc. }}\right)$ in $\mathrm{MHz}$ of the monohydrate of perillaldehyde EQ2-1w(II).

\begin{tabular}{rrrrrrrrrr}
\hline$J^{\prime}$ & $K_{a}^{\prime}$ & $K_{c}^{\prime}$ & $\leftarrow$ & $J^{\prime \prime}$ & $K_{a}^{\prime \prime}$ & $K_{c}^{\prime \prime}$ & \multicolumn{1}{c}{$v_{\text {obs. }}$} & $v_{\text {obs. }}-v_{\text {calc. }}$ & exp. error \\
\hline 6 & 0 & 6 & $\leftarrow$ & 5 & 0 & 5 & 3710.9584 & -0.0006 & 0.002 \\
7 & 1 & 7 & $\leftarrow$ & 6 & 1 & 6 & 4256.64 & -0.0006 & 0.002 \\
7 & 0 & 7 & $\leftarrow$ & 6 & 0 & 6 & 4327.2057 & -0.0002 & 0.002 \\
7 & 1 & 6 & $\leftarrow$ & 6 & 1 & 5 & 4410.478 & 0.0001 & 0.002 \\
8 & 1 & 8 & $\leftarrow$ & 7 & 1 & 7 & 4864.0159 & 0.0002 & 0.002 \\
8 & 0 & 8 & $\leftarrow$ & 7 & 0 & 7 & 4942.4291 & -0.0006 & 0.002 \\
8 & 2 & 7 & $\leftarrow$ & 7 & 2 & 6 & 4953.0627 & 0.0021 & 0.002 \\
8 & 2 & 6 & $\leftarrow$ & 7 & 2 & 5 & 4965.4834 & -0.0016 & 0.002 \\
9 & 1 & 9 & $\leftarrow$ & 8 & 1 & 8 & 5471.117 & 0.0002 & 0.001 \\
9 & 0 & 9 & $\leftarrow$ & 8 & 0 & 8 & 5556.4995 & -0.0002 & 0.002 \\
9 & 2 & 8 & $\leftarrow$ & 8 & 2 & 7 & 5571.5527 & 0.0009 & 0.002 \\
9 & 1 & 8 & $\leftarrow$ & 8 & 1 & 7 & 5668.715 & 0.0004 & 0.002 \\
10 & 1 & 10 & $\leftarrow$ & 9 & 1 & 9 & 6077.9175 & 0.0004 & 0.002 \\
10 & 0 & 10 & $\leftarrow$ & 9 & 0 & 9 & 6169.2957 & -0.0007 & 0.002 \\
10 & 2 & 9 & $\leftarrow$ & 9 & 2 & 8 & 6189.8172 & 0.0002 & 0.002 \\
10 & 2 & 8 & $\leftarrow$ & 9 & 2 & 7 & 6214.0598 & -0.0015 & 0.002 \\
10 & 1 & 9 & $\leftarrow$ & 9 & 1 & 8 & 6297.293 & 0.0004 & 0.002 \\
11 & 1 & 11 & $\leftarrow$ & 10 & 1 & 10 & 6684.3935 & 0.0008 & 0.002 \\
11 & 0 & 11 & $\leftarrow$ & 10 & 0 & 10 & 6780.7145 & -0.0009 & 0.002 \\
11 & 2 & 10 & $\leftarrow$ & 10 & 2 & 9 & 6807.8309 & -0.0003 & 0.002 \\
11 & 2 & 9 & $\leftarrow$ & 10 & 2 & 8 & 6839.992 & 0.0001 & 0.002 \\
11 & 1 & 10 & $\leftarrow$ & 10 & 1 & 9 & 6925.4425 & 0.0006 & 0.002 \\
12 & 1 & 12 & $\leftarrow$ & 11 & 1 & 11 & 7290.5221 & -0.0002 & 0.002 \\
12 & 0 & 12 & $\leftarrow$ & 11 & 0 & 11 & 7390.6715 & -0.0005 & 0.002 \\
12 & 2 & 11 & $\leftarrow$ & 11 & 2 & 10 & 7425.5698 & -0.0002 & 0.002 \\
12 & 2 & 10 & $\leftarrow$ & 11 & 2 & 9 & 7467.1013 & -0.0007 & 0.002 \\
12 & 1 & 11 & $\leftarrow$ & 11 & 1 & 10 & 7553.1083 & 0.0010 & 0.002 \\
13 & 1 & 13 & $\leftarrow$ & 12 & 1 & 12 & 7896.2887 & 0.0009 & 0.002 \\
13 & 0 & 13 & $\leftarrow$ & 12 & 0 & 12 & 7999.1047 & -0.0006 & 0.002 \\
\hline & & & & & & & & &
\end{tabular}


Table S17: Measured frequencies ( $\left.v_{\text {obs. }}\right)$ and residuals $\left(v_{\text {obs. }}-v_{\text {calc. }}\right)$ in $\mathrm{MHz}$ of the monohydrate of perillaldehyde EQ2-1w(II) labeled with ${ }^{18} \mathrm{O}$.

\begin{tabular}{rrrlrrrrrr}
\hline$J^{\prime}$ & $K_{a}^{\prime}$ & $K_{c}^{\prime}$ & $\leftarrow$ & $J^{\prime \prime}$ & $K^{\prime \prime}{ }_{a}$ & $K^{\prime \prime}{ }_{c}$ & $v_{\text {obs. }}$ & $v_{\text {obs. }}-v_{\text {calc. }}$ & exp. error \\
\hline 5 & 1 & 4 & $\leftarrow$ & 4 & 1 & 3 & 3029.6569 & 0.0004 & 0.002 \\
6 & 1 & 6 & $\leftarrow$ & 5 & 1 & 5 & 3512.3711 & -0.0008 & 0.002 \\
6 & 0 & 6 & $\leftarrow$ & 5 & 0 & 5 & 3570.3186 & 0.0004 & 0.002 \\
7 & 1 & 7 & $\leftarrow$ & 6 & 1 & 6 & 4097.2886 & 0.0000 & 0.002 \\
7 & 0 & 7 & $\leftarrow$ & 6 & 0 & 6 & 4163.42 & -0.0005 & 0.002 \\
7 & 1 & 6 & $\leftarrow$ & 6 & 1 & 5 & 4240.579 & -0.0004 & 0.002 \\
8 & 1 & 8 & $\leftarrow$ & 7 & 1 & 7 & 4681.992 & 0.0008 & 0.002 \\
8 & 0 & 8 & $\leftarrow$ & 7 & 0 & 7 & 4755.6326 & -0.0002 & 0.002 \\
8 & 1 & 7 & $\leftarrow$ & 7 & 1 & 6 & 4845.6954 & 0.0000 & 0.002 \\
9 & 1 & 9 & $\leftarrow$ & 8 & 1 & 8 & 5266.4541 & -0.0001 & 0.002 \\
9 & 0 & 9 & $\leftarrow$ & 8 & 0 & 8 & 5346.84 & 0.0003 & 0.002 \\
10 & 1 & 10 & $\leftarrow$ & 9 & 1 & 9 & 5850.6543 & 0.0002 & 0.002 \\
10 & 0 & 10 & $\leftarrow$ & 9 & 0 & 9 & 5936.9342 & 0.0001 & 0.002 \\
10 & 1 & 9 & $\leftarrow$ & 9 & 1 & 8 & 6055.0366 & 0.0000 & 0.002 \\
11 & 1 & 11 & $\leftarrow$ & 10 & 1 & 10 & 6434.5685 & -0.0003 & 0.002 \\
11 & 0 & 11 & $\leftarrow$ & 10 & 0 & 10 & 6525.8205 & -0.0001 & 0.002 \\
11 & 1 & 10 & $\leftarrow$ & 10 & 1 & 9 & 6659.1756 & 0.0000 & 0.002 \\
\hline & & & & & & & & & \\
\hline
\end{tabular}


Table S18: Measured frequencies $\left(v_{\text {obs. }}\right)$ and residuals $\left(v_{\text {obs. }}-v_{\text {calc. }}\right)$ in $\mathrm{MHz}$ of the dihydrate of perillaldehyde EQ1-2w(I).

\begin{tabular}{rrrrrrrrrr}
\hline$J^{\prime}$ & $K_{a}^{\prime}$ & $K_{c}^{\prime}$ & $\leftarrow$ & $J^{\prime \prime}$ & $K_{a}^{\prime \prime}$ & $K^{\prime \prime}{ }_{c}$ & $v_{\text {obs. }}$ & $v_{\text {obs. }}-v_{\text {calc. }}$ & exp. error \\
\hline 6 & 0 & 6 & $\leftarrow$ & 5 & 0 & 5 & 2897.2858 & 0.0002 & 0.002 \\
6 & 1 & 5 & $\leftarrow$ & 5 & 1 & 4 & 2956.3584 & -0.0007 & 0.002 \\
7 & 1 & 7 & $\leftarrow$ & 6 & 1 & 6 & 3320.8391 & -0.0006 & 0.002 \\
7 & 0 & 7 & $\leftarrow$ & 6 & 0 & 6 & 3377.7283 & 0.0002 & 0.002 \\
7 & 2 & 6 & $\leftarrow$ & 6 & 2 & 5 & 3385.515 & 0.0021 & 0.002 \\
7 & 2 & 5 & $\leftarrow$ & 6 & 2 & 4 & 3394.5472 & -0.0016 & 0.002 \\
7 & 1 & 6 & $\leftarrow$ & 6 & 1 & 5 & 3448.4373 & -0.0001 & 0.002 \\
8 & 1 & 8 & $\leftarrow$ & 7 & 1 & 7 & 3794.4757 & -0.0014 & 0.002 \\
8 & 0 & 8 & $\leftarrow$ & 7 & 0 & 7 & 3857.0701 & -0.0004 & 0.002 \\
8 & 2 & 7 & $\leftarrow$ & 7 & 2 & 6 & 3868.6121 & 0.0013 & 0.002 \\
9 & 1 & 9 & $\leftarrow$ & 8 & 1 & 8 & 4267.824 & -0.0010 & 0.002 \\
9 & 0 & 9 & $\leftarrow$ & 8 & 0 & 8 & 4335.1842 & -0.0001 & 0.002 \\
9 & 2 & 8 & $\leftarrow$ & 8 & 2 & 7 & 4351.4912 & 0.0009 & 0.002 \\
9 & 2 & 7 & $\leftarrow$ & 8 & 2 & 6 & 4370.6979 & 0.0000 & 0.002 \\
9 & 1 & 8 & $\leftarrow$ & 8 & 1 & 7 & 4431.6035 & -0.0009 & 0.002 \\
10 & 1 & 10 & $\leftarrow$ & 9 & 1 & 9 & 4740.8583 & 0.0003 & 0.002 \\
10 & 0 & 10 & $\leftarrow$ & 9 & 0 & 9 & 4811.9604 & -0.0001 & 0.002 \\
10 & 2 & 9 & $\leftarrow$ & 9 & 2 & 8 & 4834.1254 & 0.0009 & 0.002 \\
10 & 2 & 8 & $\leftarrow$ & 9 & 2 & 7 & 4860.3644 & 0.0013 & 0.002 \\
10 & 1 & 9 & $\leftarrow$ & 9 & 1 & 8 & 4922.581 & -0.0007 & 0.002 \\
11 & 1 & 11 & $\leftarrow$ & 10 & 1 & 10 & 5213.5537 & -0.0002 & 0.002 \\
11 & 0 & 11 & $\leftarrow$ & 10 & 0 & 10 & 5287.317 & 0.0001 & 0.002 \\
11 & 2 & 10 & $\leftarrow$ & 10 & 2 & 9 & 5316.4875 & 0.0009 & 0.002 \\
11 & 2 & 9 & $\leftarrow$ & 10 & 2 & 8 & 5351.1692 & -0.0001 & 0.002 \\
11 & 1 & 10 & $\leftarrow$ & 10 & 1 & 9 & 5413.0733 & -0.0009 & 0.002 \\
12 & 1 & 12 & $\leftarrow$ & 11 & 1 & 11 & 5685.8948 & -0.0003 & 0.002 \\
12 & 0 & 12 & $\leftarrow$ & 11 & 0 & 11 & 5761.2042 & 0.0010 & 0.002 \\
12 & 2 & 11 & $\leftarrow$ & 11 & 2 & 10 & 5798.5502 & 0.0000 & 0.002 \\
12 & 3 & 10 & $\leftarrow$ & 11 & 3 & 9 & 5811.1357 & 0.0001 & 0.002 \\
\hline & & & & & & & & &
\end{tabular}




\begin{tabular}{rrrlrrrrrr}
\hline$J^{\prime}$ & $K_{a}^{\prime}$ & $K_{c}^{\prime}$ & $\leftarrow$ & $J^{\prime \prime}$ & $K^{\prime \prime}{ }_{a}$ & $K_{c}^{\prime \prime}$ & $v_{\text {obs. }}$ & $v_{\text {obs. }}-v_{\text {calc. }}$ & exp. error \\
\hline 12 & 2 & 10 & $\leftarrow$ & 11 & 2 & 9 & 5843.1393 & 0.0012 & 0.002 \\
12 & 1 & 11 & $\leftarrow$ & 11 & 1 & 10 & 5903.015 & -0.0004 & 0.002 \\
13 & 1 & 13 & $\leftarrow$ & 12 & 1 & 12 & 6157.8671 & 0.0000 & 0.002 \\
13 & 0 & 13 & $\leftarrow$ & 12 & 0 & 12 & 6233.6077 & 0.0006 & 0.002 \\
13 & 2 & 12 & $\leftarrow$ & 12 & 2 & 11 & 6280.289 & -0.0001 & 0.002 \\
13 & 3 & 10 & $\leftarrow$ & 12 & 3 & 9 & 6298.7969 & -0.0022 & 0.002 \\
13 & 2 & 11 & $\leftarrow$ & 12 & 2 & 10 & 6336.2527 & 0.0005 & 0.002 \\
13 & 1 & 12 & $\leftarrow$ & 12 & 1 & 11 & 6392.3341 & -0.0002 & 0.002 \\
14 & 1 & 14 & $\leftarrow$ & 13 & 1 & 13 & 6629.4594 & -0.0001 & 0.002 \\
14 & 0 & 14 & $\leftarrow$ & 13 & 0 & 13 & 6704.5581 & 0.0009 & 0.002 \\
14 & 2 & 13 & $\leftarrow$ & 13 & 2 & 12 & 6761.6749 & -0.0030 & 0.002 \\
14 & 3 & 11 & $\leftarrow$ & 13 & 3 & 10 & 6785.0984 & 0.0014 & 0.002 \\
14 & 2 & 12 & $\leftarrow$ & 13 & 2 & 11 & 6830.4544 & 0.0012 & 0.002 \\
14 & 1 & 13 & $\leftarrow$ & 13 & 1 & 12 & 6880.9545 & 0.0000 & 0.002 \\
15 & 1 & 15 & $\leftarrow$ & 14 & 1 & 14 & 7100.6657 & -0.0007 & 0.002 \\
15 & 0 & 15 & $\leftarrow$ & 14 & 0 & 14 & 7174.124 & 0.0013 & 0.002 \\
15 & 2 & 14 & $\leftarrow$ & 14 & 2 & 13 & 7242.6906 & -0.0009 & 0.002 \\
\hline & & & & & & & & &
\end{tabular}


Table S19: Measured frequencies $\left(v_{\mathrm{obs}}\right)$ and residuals $\left(v_{\mathrm{obs} .}-v_{\text {calc. }}\right)$ in $\mathrm{MHz}$ of the dihydrate of perillaldehyde EQ1-2w(I) with the ${ }^{16} \mathrm{O}_{\mathrm{w} 1}$ atom substituted by a ${ }^{18} \mathrm{O}$ atom.

\begin{tabular}{rrrrrrrrrr}
\hline$J^{\prime}$ & $K_{a}^{\prime}$ & $K_{c}^{\prime}$ & $\leftarrow$ & $J^{\prime \prime}$ & $K^{\prime \prime}{ }_{a}$ & $K^{\prime \prime}{ }_{c}$ & $v_{\text {obs. }}$ & $v_{\text {obs. }}-v_{\text {calc. }}$ & exp. error \\
\hline 7 & 0 & 7 & $\leftarrow$ & 6 & 0 & 6 & 3288.6355 & 0.0000 & 0.002 \\
8 & 0 & 8 & $\leftarrow$ & 7 & 0 & 7 & 3755.5439 & 0.0000 & 0.002 \\
8 & 2 & 7 & $\leftarrow$ & 7 & 2 & 6 & 3766.0146 & -0.0003 & 0.003 \\
9 & 1 & 9 & $\leftarrow$ & 8 & 1 & 8 & 4156.5129 & -0.0007 & 0.002 \\
9 & 0 & 9 & $\leftarrow$ & 8 & 0 & 8 & 4221.3326 & 0.0000 & 0.002 \\
9 & 2 & 8 & $\leftarrow$ & 8 & 2 & 7 & 4236.1405 & 0.0060 & 0.005 \\
10 & 1 & 10 & $\leftarrow$ & 9 & 1 & 9 & 4617.2825 & 0.0002 & 0.002 \\
10 & 2 & 9 & $\leftarrow$ & 9 & 2 & 8 & 4706.0314 & 0.0001 & 0.002 \\
10 & 1 & 9 & $\leftarrow$ & 9 & 1 & 8 & 4790.4182 & 0.0005 & 0.003 \\
11 & 2 & 10 & $\leftarrow$ & 10 & 2 & 9 & 5175.6803 & -0.0008 & 0.002 \\
11 & 1 & 10 & $\leftarrow$ & 10 & 1 & 9 & 5267.8661 & -0.0001 & 0.002 \\
12 & 1 & 12 & $\leftarrow$ & 11 & 1 & 11 & 5537.8771 & 0.0003 & 0.002 \\
\hline
\end{tabular}

Table S20: Measured frequencies $\left(v_{\mathrm{obs}}\right)$ and residuals $\left(v_{\mathrm{obs} .}-v_{\text {calc. }}\right)$ in $\mathrm{MHz}$ of the dihydrate of perillaldehyde EQ1-2w(I) with the ${ }^{16} \mathrm{O}_{\mathrm{w} 2}$ atom substituted by a ${ }^{18} \mathrm{O}$ atom.

\begin{tabular}{rrrrrrrrrrr}
\hline$J^{\prime}$ & $K_{a}^{\prime}$ & $K_{c}^{\prime}$ & $\leftarrow$ & $J^{\prime \prime}$ & $K^{\prime \prime}{ }_{a}$ & $K^{\prime \prime}{ }_{c}$ & $v_{\text {obs. }}$ & $v_{\text {obs. }}-v_{\text {calc. }}$ & exp. error \\
\hline 8 & 0 & 8 & $\leftarrow$ & 7 & 0 & 7 & 3777.1192 & 0.0008 & 0.002 \\
8 & 1 & 7 & $\leftarrow$ & 7 & 1 & 6 & 3861.3323 & -0.0003 & 0.002 \\
9 & 0 & 9 & $\leftarrow$ & 8 & 0 & 8 & 4245.0576 & -0.0011 & 0.002 \\
9 & 2 & 8 & $\leftarrow$ & 8 & 2 & 7 & 4262.1147 & 0.0000 & 0.002 \\
9 & 1 & 8 & $\leftarrow$ & 8 & 1 & 7 & 4342.8185 & 0.0000 & 0.002 \\
10 & 1 & 10 & $\leftarrow$ & 9 & 1 & 9 & 4640.6579 & 0.0001 & 0.002 \\
10 & 0 & 10 & $\leftarrow$ & 9 & 0 & 9 & 4711.6086 & 0.0003 & 0.002 \\
10 & 1 & 9 & $\leftarrow$ & 9 & 1 & 8 & 4823.8592 & 0.0002 & 0.002 \\
11 & 1 & 11 & $\leftarrow$ & 10 & 1 & 10 & 5103.2754 & -0.0002 & 0.002 \\
\hline
\end{tabular}


Table S21: Measured frequencies $\left(v_{\mathrm{obs}}\right)$ and residuals $\left(v_{\mathrm{obs} .}-v_{\text {calc. }}\right)$ in $\mathrm{MHz}$ of the dihydrate of perillaldehyde EQ1-2w(I) with the ${ }^{16} \mathrm{O}_{\mathrm{w} 1}$ and ${ }^{16} \mathrm{O}_{\mathrm{w} 2}$ atoms substituted by ${ }^{18} \mathrm{O}$ atoms.

\begin{tabular}{rrrrrrrrrr}
\hline$J^{\prime}$ & $K_{a}^{\prime}$ & $K_{c}^{\prime}$ & $\leftarrow$ & $J^{\prime \prime}$ & $K^{\prime \prime}{ }_{a}$ & $K^{\prime \prime}{ }_{c}$ & $v_{\text {obs. }}$ & $v_{\text {obs. }}-v_{\text {calc. }}$ & exp. error \\
\hline 6 & 1 & 5 & $\leftarrow$ & 5 & 1 & 4 & 2822.2391 & -0.0005 & 0.002 \\
7 & 1 & 7 & $\leftarrow$ & 6 & 1 & 6 & 3169.073 & 0.0000 & 0.002 \\
7 & 0 & 7 & $\leftarrow$ & 6 & 0 & 6 & 3223.9292 & -0.0007 & 0.002 \\
7 & 1 & 6 & $\leftarrow$ & 6 & 1 & 5 & 3291.9935 & -0.0002 & 0.002 \\
8 & 0 & 8 & $\leftarrow$ & 7 & 0 & 7 & 3681.4424 & 0.0000 & 0.002 \\
8 & 1 & 7 & $\leftarrow$ & 7 & 1 & 6 & 3761.4506 & 0.0005 & 0.002 \\
9 & 0 & 9 & $\leftarrow$ & 8 & 0 & 8 & 4137.7801 & 0.0001 & 0.002 \\
9 & 2 & 7 & $\leftarrow$ & 8 & 2 & 6 & 4171.7197 & 0.0000 & 0.002 \\
9 & 1 & 8 & $\leftarrow$ & 8 & 1 & 7 & 4230.557 & -0.0001 & 0.002 \\
10 & 1 & 10 & $\leftarrow$ & 9 & 1 & 9 & 4524.1891 & 0.0000 & 0.002 \\
10 & 0 & 10 & $\leftarrow$ & 9 & 0 & 9 & 4592.8391 & 0.0014 & 0.002 \\
10 & 1 & 9 & $\leftarrow$ & 9 & 1 & 8 & 4699.2604 & 0.0001 & 0.002 \\
12 & 0 & 12 & $\leftarrow$ & 11 & 0 & 11 & 5498.8242 & -0.0008 & 0.002 \\
\hline
\end{tabular}


Table S22: Measured frequencies $\left(v_{\text {obs. }}\right)$ and residuals $\left(v_{\text {obs. }}-v_{\text {calc. }}\right)$ in $\mathrm{MHz}$ of the dihydrate of perillaldehyde EQ2-2w(I).

\begin{tabular}{rrrrrrrrrr}
\hline$J^{\prime}$ & $K_{a}^{\prime}$ & $K_{c}^{\prime}$ & $\leftarrow$ & $J^{\prime \prime}$ & $K_{a}^{\prime \prime}$ & $K_{c}^{\prime \prime}$ & $v_{\text {obs. }}$ & $v_{\text {obs. }}-v_{\text {calc. }}$ & exp. error \\
\hline 5 & 1 & 5 & $\leftarrow$ & 4 & 1 & 4 & 2352.2052 & -0.0001 & 0.002 \\
5 & 0 & 5 & $\leftarrow$ & 4 & 0 & 4 & 2408.1278 & 0.0005 & 0.002 \\
5 & 1 & 4 & $\leftarrow$ & 4 & 1 & 3 & 2473.0737 & -0.0002 & 0.001 \\
6 & 1 & 6 & $\leftarrow$ & 5 & 1 & 5 & 2821.8801 & -0.0001 & 0.002 \\
6 & 1 & 5 & $\leftarrow$ & 5 & 1 & 4 & 2966.8547 & 0.0001 & 0.001 \\
7 & 1 & 7 & $\leftarrow$ & 6 & 1 & 6 & 3291.156 & -0.0004 & 0.002 \\
7 & 0 & 7 & $\leftarrow$ & 6 & 0 & 6 & 3363.3814 & 0.0000 & 0.002 \\
7 & 2 & 6 & $\leftarrow$ & 6 & 2 & 5 & 3377.2199 & 0.0017 & 0.002 \\
7 & 1 & 6 & $\leftarrow$ & 6 & 1 & 5 & 3460.1605 & 0.0003 & 0.002 \\
8 & 1 & 8 & $\leftarrow$ & 7 & 1 & 7 & 3759.9833 & 0.0000 & 0.002 \\
8 & 0 & 8 & $\leftarrow$ & 7 & 0 & 7 & 3838.2567 & 0.0001 & 0.002 \\
8 & 2 & 7 & $\leftarrow$ & 7 & 2 & 6 & 3858.7075 & 0.0003 & 0.002 \\
8 & 2 & 6 & $\leftarrow$ & 7 & 2 & 5 & 3882.6736 & -0.0002 & 0.002 \\
8 & 1 & 7 & $\leftarrow$ & 7 & 1 & 6 & 3952.8949 & 0.0001 & 0.002 \\
9 & 1 & 9 & $\leftarrow$ & 8 & 1 & 8 & 4228.3171 & 0.0003 & 0.002 \\
9 & 0 & 9 & $\leftarrow$ & 8 & 0 & 8 & 4311.0359 & -0.0003 & 0.002 \\
9 & 2 & 8 & $\leftarrow$ & 8 & 2 & 7 & 4339.809 & 0.0000 & 0.002 \\
9 & 1 & 8 & $\leftarrow$ & 8 & 1 & 7 & 4444.9547 & 0.0001 & 0.002 \\
10 & 1 & 10 & $\leftarrow$ & 9 & 1 & 9 & 4696.1205 & 0.0001 & 0.001 \\
10 & 0 & 10 & $\leftarrow$ & 9 & 0 & 9 & 4781.5932 & -0.0003 & 0.001 \\
10 & 2 & 9 & $\leftarrow$ & 9 & 2 & 8 & 4820.4761 & 0.0000 & 0.001 \\
10 & 3 & 8 & $\leftarrow$ & 9 & 3 & 7 & 4833.4818 & -0.0011 & 0.002 \\
10 & 2 & 8 & $\leftarrow$ & 9 & 2 & 7 & 4866.6227 & -0.0004 & 0.002 \\
10 & 1 & 9 & $\leftarrow$ & 9 & 1 & 8 & 4936.2267 & 0.0000 & 0.002 \\
11 & 1 & 11 & $\leftarrow$ & 10 & 1 & 10 & 5163.3656 & 0.0000 & 0.002 \\
11 & 0 & 11 & $\leftarrow$ & 10 & 0 & 10 & 5249.8739 & 0.0000 & 0.002 \\
11 & 2 & 10 & $\leftarrow$ & 10 & 2 & 9 & 5300.6623 & 0.0002 & 0.002 \\
12 & 1 & 12 & $\leftarrow$ & 11 & 1 & 11 & 5630.0319 & -0.0001 & 0.002 \\
12 & 0 & 12 & $\leftarrow$ & 11 & 0 & 11 & 5715.9053 & 0.0000 & 0.002 \\
\hline
\end{tabular}




\begin{tabular}{rrrlrrrrrr}
\hline$J^{\prime}$ & $K_{a}^{\prime}$ & $K_{c}^{\prime}$ & $\leftarrow$ & $J^{\prime \prime}$ & $K^{\prime \prime}{ }_{a}$ & $K^{\prime \prime}{ }_{c}$ & $v_{\text {obs. }}$ & $v_{\text {obs. }}-v_{\text {calc. }}$ & exp. error \\
\hline 13 & 1 & 13 & $\leftarrow$ & 12 & 1 & 12 & 6096.1078 & 0.0002 & 0.002 \\
13 & 2 & 12 & $\leftarrow$ & 12 & 2 & 11 & 6259.4093 & 0.0011 & 0.002 \\
13 & 2 & 11 & $\leftarrow$ & 12 & 2 & 10 & 6355.4943 & -0.0007 & 0.002 \\
13 & 1 & 12 & $\leftarrow$ & 12 & 1 & 11 & 6404.0394 & 0.0001 & 0.002 \\
14 & 1 & 14 & $\leftarrow$ & 13 & 1 & 13 & 6561.5884 & 0.0000 & 0.002 \\
14 & 0 & 14 & $\leftarrow$ & 13 & 0 & 13 & 6641.7486 & -0.0008 & 0.002 \\
14 & 2 & 13 & $\leftarrow$ & 13 & 2 & 12 & 6737.8814 & 0.0006 & 0.002 \\
\hline
\end{tabular}

Table S23: Measured frequencies $\left(v_{\mathrm{obs}}\right)$ and residuals $\left(v_{\mathrm{obs}}-v_{\text {calc. }}\right)$ in $\mathrm{MHz}$ of the dihydrate of perillaldehyde EQ2-2w(I) with the ${ }^{16} \mathrm{O}_{\mathrm{w} 1}$ atom substituted by a ${ }^{18} \mathrm{O}$ atom.

\begin{tabular}{rrrrrrrrrr}
\hline$J^{\prime}$ & $K_{a}^{\prime}$ & $K_{c}^{\prime}$ & $\leftarrow$ & $J^{\prime \prime}$ & $K^{\prime \prime}{ }_{a}$ & $K^{\prime \prime}{ }_{c}$ & $v_{\text {obs. }}$ & $v_{\text {obs. }}-v_{\text {calc. }}$ & exp. error \\
\hline 7 & 1 & 7 & $\leftarrow$ & 6 & 1 & 6 & 3205.7878 & -0.0004 & 0.002 \\
7 & 0 & 7 & $\leftarrow$ & 6 & 0 & 6 & 3275.0146 & 0.0001 & 0.002 \\
8 & 1 & 8 & $\leftarrow$ & 7 & 1 & 7 & 3662.546 & 0.0003 & 0.002 \\
8 & 2 & 7 & $\leftarrow$ & 7 & 2 & 6 & 3756.2737 & 0.0000 & 0.002 \\
8 & 1 & 7 & $\leftarrow$ & 7 & 1 & 6 & 3845.9086 & 0.0000 & 0.002 \\
10 & 1 & 9 & $\leftarrow$ & 9 & 1 & 8 & 4802.9979 & -0.0001 & 0.002 \\
11 & 0 & 11 & $\leftarrow$ & 10 & 0 & 10 & 5114.262 & 0.0048 & 0.005 \\
11 & 1 & 10 & $\leftarrow$ & 10 & 1 & 9 & 5280.3782 & 0.0001 & 0.002 \\
12 & 1 & 12 & $\leftarrow$ & 11 & 1 & 11 & 5484.7769 & 0.0000 & 0.002 \\
12 & 0 & 12 & $\leftarrow$ & 11 & 0 & 11 & 5568.8975 & -0.0011 & 0.002 \\
13 & 0 & 13 & $\leftarrow$ & 12 & 0 & 12 & 6021.5218 & 0.0003 & 0.002 \\
\hline
\end{tabular}


Table S24: Measured frequencies $\left(v_{\mathrm{obs}}\right)$ and residuals $\left(v_{\mathrm{obs} .}-v_{\text {calc. }}\right)$ in $\mathrm{MHz}$ of the dihydrate of perillaldehyde EQ2-2w(I) with the ${ }^{16} \mathrm{O}_{\mathrm{w} 2}$ atom substituted by a ${ }^{18} \mathrm{O}$ atom.

\begin{tabular}{rrrrrrrrrrr}
\hline$J^{\prime}$ & $K_{a}^{\prime}$ & $K_{c}^{\prime}$ & $\leftarrow$ & $J^{\prime \prime}$ & $K_{a}^{\prime \prime}$ & $K^{\prime \prime}{ }_{c}$ & $v_{\text {obs. }}$ & $v_{\text {obs. }}-v_{\text {calc. }}$ & exp. error \\
\hline 8 & 1 & 8 & $\leftarrow$ & 7 & 1 & 7 & 3681.8471 & 0.0000 & 0.002 \\
8 & 0 & 8 & $\leftarrow$ & 7 & 0 & 7 & 3759.3552 & 0.0000 & 0.002 \\
9 & 1 & 9 & $\leftarrow$ & 8 & 1 & 8 & 4140.3809 & 0.0000 & 0.002 \\
9 & 0 & 9 & $\leftarrow$ & 8 & 0 & 8 & 4222.134 & 0.0000 & 0.002 \\
9 & 2 & 8 & $\leftarrow$ & 8 & 2 & 7 & 4251.516 & 0.0001 & 0.002 \\
9 & 1 & 8 & $\leftarrow$ & 8 & 1 & 7 & 4356.1622 & 0.0000 & 0.002 \\
10 & 1 & 10 & $\leftarrow$ & 9 & 1 & 9 & 4598.3761 & 0.0000 & 0.002 \\
10 & 2 & 9 & $\leftarrow$ & 9 & 2 & 8 & 4722.3389 & 0.0000 & 0.002 \\
11 & 2 & 10 & $\leftarrow$ & 10 & 2 & 9 & 5192.67 & -0.0002 & 0.002 \\
12 & 2 & 11 & $\leftarrow$ & 11 & 2 & 10 & 5662.4634 & 0.0001 & 0.002 \\
\hline
\end{tabular}

Table S25: Measured frequencies $\left(v_{\mathrm{obs}}\right)$ and residuals $\left(v_{\mathrm{obs} .}-v_{\text {calc. }}\right)$ in $\mathrm{MHz}$ of the dihydrate of perillaldehyde EQ2-2w(I) with the ${ }^{16} \mathrm{O}_{\mathrm{w} 1}$ and ${ }^{16} \mathrm{O}_{\mathrm{w} 2}$ atoms substituted by ${ }^{18} \mathrm{O}$ atoms.

\begin{tabular}{rrrrrrrrrr}
\hline$J^{\prime}$ & $K_{a}^{\prime}$ & $K_{c}^{\prime}$ & $\leftarrow$ & $J^{\prime \prime}$ & $K_{a}^{\prime \prime}$ & $K^{\prime \prime}{ }_{c}$ & $v_{\text {obs. }}$ & $v_{\text {obs. }}-v_{\text {calc. }}$ & exp. error \\
\hline 8 & 0 & 8 & $\leftarrow$ & 7 & 0 & 7 & 3664.6377 & -0.0002 & 0.002 \\
8 & 1 & 7 & $\leftarrow$ & 7 & 1 & 6 & 3773.1035 & -0.0007 & 0.002 \\
9 & 0 & 9 & $\leftarrow$ & 8 & 0 & 8 & 4116.2052 & 0.0001 & 0.002 \\
9 & 2 & 8 & $\leftarrow$ & 8 & 2 & 7 & 4142.9358 & 0.0005 & 0.002 \\
9 & 1 & 8 & $\leftarrow$ & 8 & 1 & 7 & 4242.8418 & 0.0002 & 0.002 \\
10 & 2 & 9 & $\leftarrow$ & 9 & 2 & 8 & 4601.8378 & 0.0001 & 0.002 \\
10 & 1 & 9 & $\leftarrow$ & 9 & 1 & 8 & 4711.8515 & 0.0008 & 0.002 \\
11 & 1 & 11 & $\leftarrow$ & 10 & 1 & 10 & 4930.0184 & -0.0002 & 0.002 \\
11 & 2 & 10 & $\leftarrow$ & 10 & 2 & 9 & 5060.2927 & -0.0006 & 0.002 \\
11 & 1 & 10 & $\leftarrow$ & 10 & 1 & 9 & 5180.0184 & -0.0003 & 0.002 \\
12 & 1 & 12 & $\leftarrow$ & 11 & 1 & 11 & 5375.6579 & 0.0002 & 0.002 \\
\hline
\end{tabular}


Table S26: The computed rotational constants and dipole moment components of the two trihydrates of perillaldehyde

\begin{tabular}{lrrrr}
\hline & \multicolumn{2}{c}{ EQ1-3w } & \multicolumn{2}{c}{ EQ2-3w } \\
\hline$A / \mathrm{MHz}{ }^{a}$ & $\mathrm{DFT}^{b}$ & $\mathrm{MP2}^{a}$ & $\mathrm{DFT}^{b}$ \\
\hline $\mathrm{B} / \mathrm{MHz}$ & 1126.21 & 1150.56 & 1121.84 & 1144.21 \\
$C / \mathrm{MHz}$ & 225.94 & 228.27 & 227.52 & 229.69 \\
$\kappa^{c}$ & 199.07 & 201.57 & 196.64 & 199.46 \\
$\left|\mu_{a}\right| / \mathrm{D}$ & -0.942 & -0.942 & -0.933 & -0.936 \\
$\left|\mu_{b}\right| / \mathrm{D}$ & 0.83 & 1.44 & 0.82 & 1.45 \\
$\left|\mu_{c}\right| / \mathrm{D}$ & 0.03 & 0.28 & 0.40 & 0.68 \\
$\Delta E^{d} /\left(\mathrm{kJ} \mathrm{mol}^{-1}\right)$ & 0.00 & 0.00 & 2.13 & 1.33 \\
\hline
\end{tabular}

${ }^{a} \mathrm{MP} 2 / 6-311++\mathrm{G}(\mathrm{d}, \mathrm{p}) ;{ }^{b}$ B3LYP-D3BJ / def2-TZVP ; ${ }^{c} \kappa=(2 B-A-C) /(A-C) ;{ }^{d}$ difference of energy, including ZPE correction, with respect to the lowest energy conformer EQ1-3w.

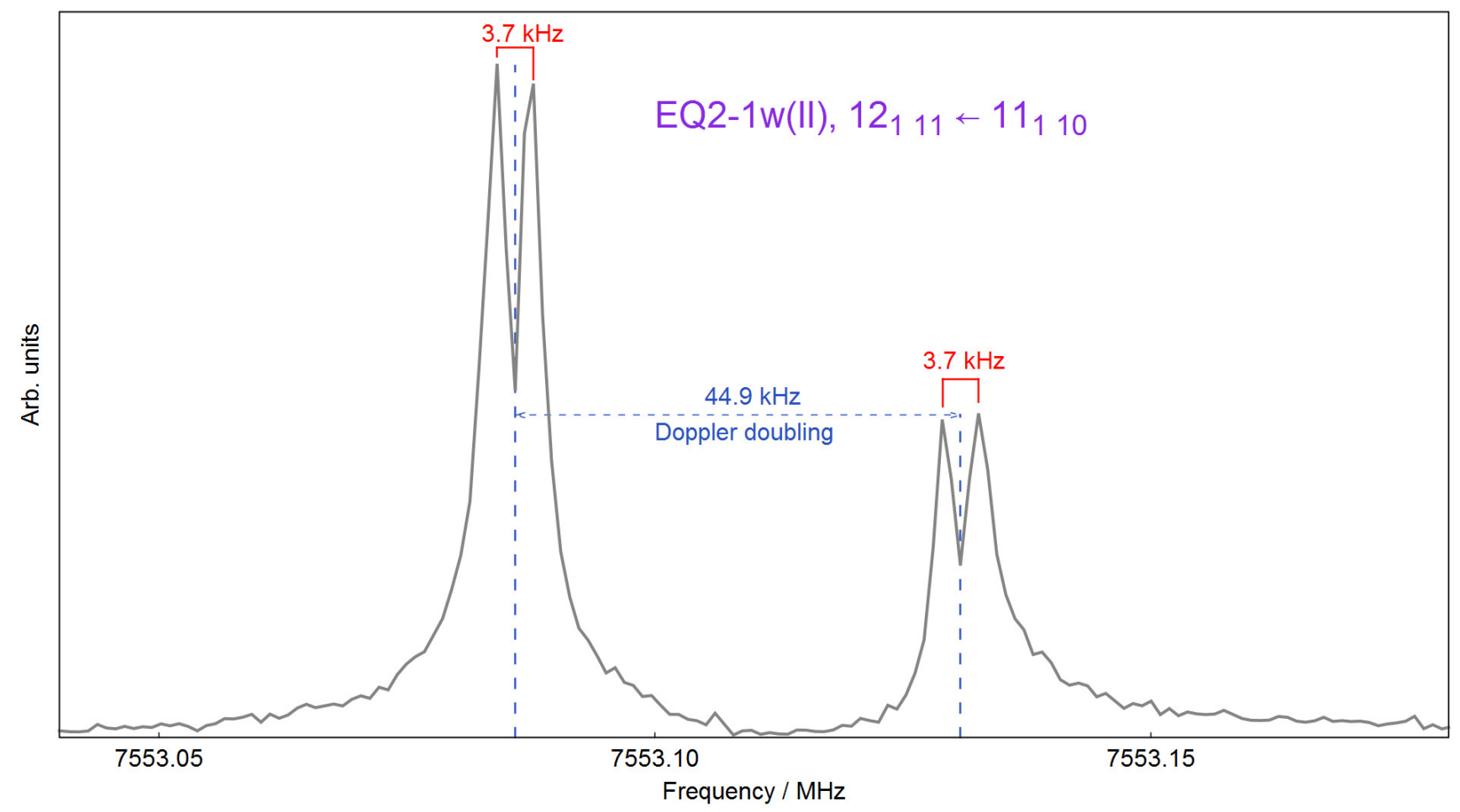

Figure S7: An example of a structured line of a monohydrate of perillaldehyde. The splitting of $3.7 \mathrm{kHz}$ in this case can be clearly seen. 


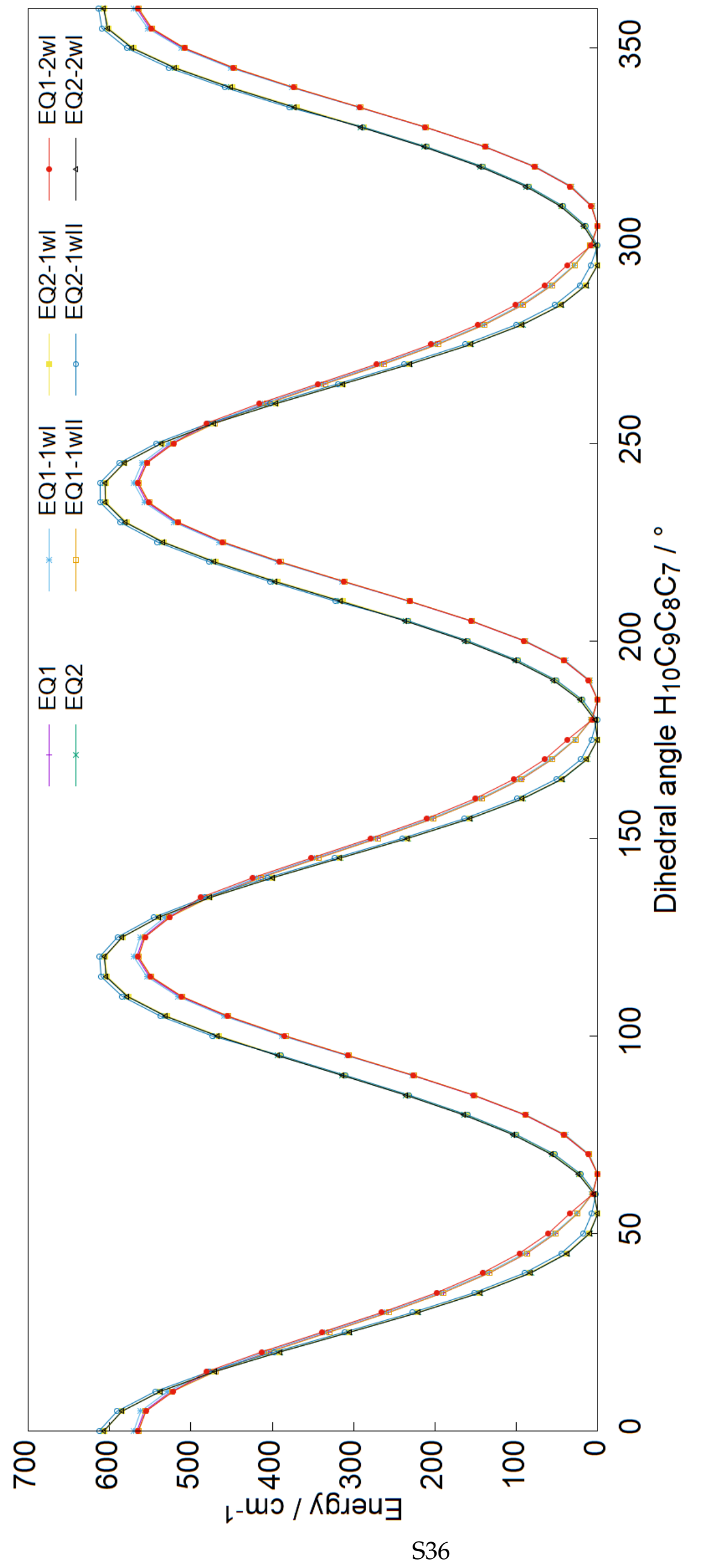

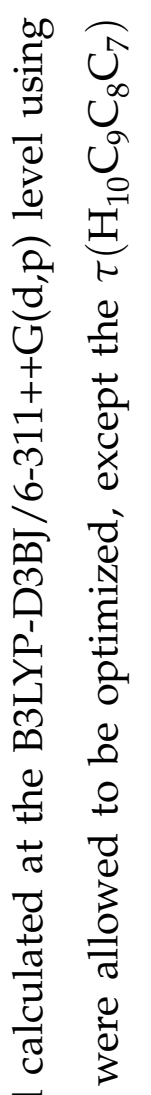

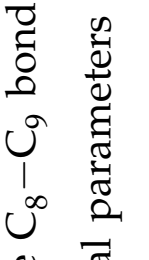

吾

卷

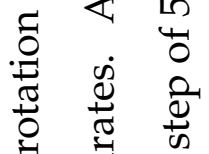

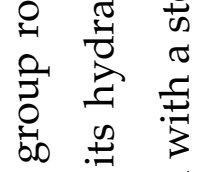

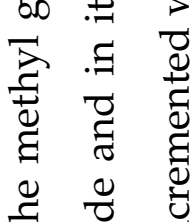

पे ह

\& $\frac{0}{0}$ 吾

可

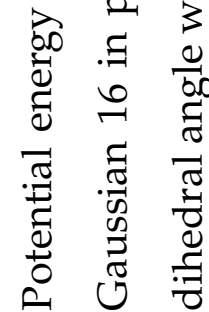

$\ddot{\infty}$

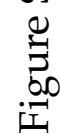

